\title{
Thermochemical and Kinetic Study of the Carbocation Ring Contraction of Cyclohexylium to Methylcyclopentylium.
}

\author{
Iain D. Mackie, ${ }^{\ddagger}$ Jagannathan Govindhakannan $^{\dagger}$ and Gino A. DiLabio ${ }^{\ddagger^{*}}$
}

National Institute for Nanotechnology, 11421 Saskatchewan Drive, Edmonton, Alberta, Canada T6G 2M9; National Centre for Upgrading Technology, Natural Resources Canada, 1 Oil Patch Drive, Devon, Alberta, Canada T9G 1A8

Table S1. Non-ZPVE corrected relative (to $\mathbf{I}_{\mathrm{f}}$ ) energies.

Table S2. ZPVE corrected relative (to $\mathbf{I}_{\mathrm{f}}$ ) energies.

Table S3. Non-ZPVE corrected 6-311++G(2d,2p) electronic energies.

Table S4. ZPVE corrected 6-311++G(2d,2p) electronic energies

Table S5. QCISD/6-311++G(d,p) optimised Cartesian coordinates $(\AA)$ of $\mathbf{I}_{\mathrm{f}}$.

Table S6. QCISD/6-311++G(d,p) optimised Cartesian coordinates ( $\AA$ ) of $\mathbf{I}_{\mathrm{p}}$.

Table S7. QCISD/6-311++G(d,p) optimised Cartesian coordinates ( $⿱$ A) of $\mathbf{I}_{\mathrm{d}}$.

Table S8. QCISD/6-311++G(d,p) optimised Cartesian coordinates ( $)$ of $\mathbf{I}_{\text {int }}$.

Table S9. QCISD/6-311++G(d,p) optimised Cartesian coordinates ( $\AA$ ) of II.

Table S10. QCISD/6-311++G(d,p) optimised Cartesian coordinates $(\AA)$ of $\mathrm{TS}_{\mathbf{I f} \rightarrow \mathrm{Ip}}$.

Table S11. QCISD/6-311++G(d,p) optimised Cartesian coordinates ( $\AA$ ) of $\mathrm{TS}_{\text {If } \rightarrow \mathbf{I d}}$.

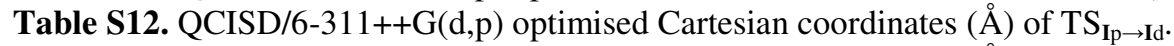

Table S13. QCISD/6-311++G(d,p) optimised Cartesian coordinates $(\AA)$ of $\mathrm{TS}_{\mathrm{I} \rightarrow \mathrm{Iint}}$.

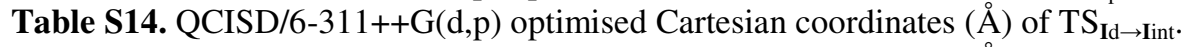

Table S15. QCISD/6-311++G(d,p) optimised Cartesian coordinates $(\AA)$ of $\mathrm{TS}_{\text {Iint } \rightarrow \mathrm{II}}$.

Table S16. B3LYP/6-311++G(2d,2p) optimised Cartesian coordinates $(\AA)$ of $\mathbf{I}_{\mathrm{f}}$.

Table S17. B3LYP /6-311++G(2d,2p) optimised Cartesian coordinates ( $⿱$ A) of $\mathbf{I}_{\mathrm{p}}$.

Table S18. B3LYP /6-311++G(2d,2p) optimised Cartesian coordinates $(\AA)$ of $\mathbf{I}_{\mathrm{d}}$.

Table S19. B3LYP /6-311++G(2d,2p) optimised Cartesian coordinates $(\AA)$ of $\mathbf{I}_{\text {int }}$.

Table S20. B3LYP /6-311++G(2d,2p) optimised Cartesian coordinates ( $\mathrm{A})$ of II.

Table S21. B3LYP /6-311++G(2d,2p) optimised Cartesian coordinates $(\AA)$ of $\mathrm{TS}_{\mathrm{If} \rightarrow \mathrm{Ip}}$.

Table S22. B3LYP /6-311++G(2d,2p) optimised Cartesian coordinates $(\AA)$ of $\mathrm{TS}_{\mathrm{If} \rightarrow \mathbf{I d} \text {. }}$.

Table S23. B3LYP /6-311++G(2d,2p) optimised Cartesian coordinates $(\AA)$ of $\mathrm{TS}_{\mathrm{Ip} \rightarrow \mathrm{Id}}$.

Table S24. B3LYP /6-311++G(2d,2p) optimised Cartesian coordinates $(\AA)$ of $\mathrm{TS}_{\mathrm{I} \mathrm{I} \rightarrow \mathrm{Iint}}$.

Table S25. B3LYP /6-311++G(2d,2p) optimised Cartesian coordinates $(\AA)$ of $\mathrm{TS}_{\mathrm{Id} \rightarrow \mathrm{Iint}}$.

Table S26. B3LYP /6-311++G(2d,2p) optimised Cartesian coordinates $(\AA)$ of $\mathrm{TS}_{\text {Iint } \rightarrow \text { II }}$.

Table S27. MP2/6-311++G(2d,2p) optimised Cartesian coordinates $(\AA)$ of $\mathbf{I}_{\mathrm{f}}$.

Table S28. MP2/6-311++G(2d,2p) optimised Cartesian coordinates $(\AA)$ of $\mathbf{I}_{\mathrm{p}}$.

Table S29. MP2/6-311++G(2d,2p) optimised Cartesian coordinates $(\AA)$ of $\mathbf{I}_{d}$.

Table S30. MP2/6-311++G(2d,2p) optimised Cartesian coordinates ( $⿱$ $)$ of $\mathbf{I}_{\text {int }}$.

Table S31. MP2/6-311++G(2d,2p) optimised Cartesian coordinates ( $⿱$ A) of II.

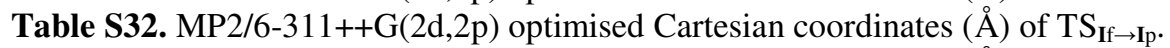

Table S33. MP2/6-311++G(2d,2p) optimised Cartesian coordinates $(\AA)$ of $\mathrm{TS}_{\text {If } \rightarrow \text { Id }}$.

Table S34. MP2/6-311++G(2d,2p) optimised Cartesian coordinates $(\AA)$ of $\mathrm{TS}_{\mathrm{Ip} \rightarrow \mathrm{Id}}$.

Table S35. MP2/6-311++G(2d,2p) optimised Cartesian coordinates $(\AA)$ of $\mathrm{TS}_{\mathrm{Ip} \rightarrow \mathrm{Int}}$.

\footnotetext{
$\ddagger$ NINT-NRC

${ }^{\dagger}$ NCUT-NRCan

* Author to whom correspondence should be addressed. Phone: +1-780-641-1729. E-mail:

Gino.DiLabio@nrc.ca
} 
Table S36. MP2/6-311++G(2d,2p) optimised Cartesian coordinates $(\AA)$ of $\mathrm{TS}_{\mathrm{Id} \rightarrow \mathrm{Iint}}$. Table S37. MP2/6-311++G(2d,2p) optimised Cartesian coordinates $(\AA)$ of $\mathrm{TS}_{\text {Int } \rightarrow \text { II }}$. Table S38. PBE/6-311++G(2d,2p) optimised Cartesian coordinates $(\AA)$ of $\mathbf{I}_{\mathrm{f}}$.

Table S39. PBE /6-311++G(2d,2p) optimised Cartesian coordinates ( $\AA$ ) of $\mathbf{I}_{\mathrm{p}}$. Table S40. PBE /6-311++G(2d,2p) optimised Cartesian coordinates ( $\AA$ ) of $\mathbf{I}_{\mathbf{d}}$. Table S41. PBE /6-311++G(2d,2p) optimised Cartesian coordinates ( $(\AA)$ of $\mathbf{I}_{\text {int }}$. Table S42. PBE /6-311++G(2d,2p) optimised Cartesian coordinates ( $\AA$ ) of II. Table S43. PBE /6-311++G(2d,2p) optimised Cartesian coordinates $(\AA)$ of $\mathrm{TS}_{\mathrm{If} \rightarrow \mathrm{I} p}$. Table S44. PBE /6-311++G(2d,2p) optimised Cartesian coordinates ( $\AA$ ) of $\mathrm{TS}_{\mathrm{If} \rightarrow \mathrm{Id}}$. Table S45. PBE /6-311++G(2d,2p) optimised Cartesian coordinates $(\AA)$ of $\mathrm{TS}_{\mathrm{Ip} \rightarrow \mathrm{Id}}$. Table S46. PBE /6-311++G(2d,2p) optimised Cartesian coordinates $(\AA)$ of $\mathrm{TS}_{\mathrm{Ip} \rightarrow \text { Iint }}$. Table S47. PBE /6-311++G(2d,2p) optimised Cartesian coordinates $(\AA)$ of $\mathrm{TS}_{\mathrm{I} d \rightarrow \mathrm{Inn}}$. Table S48. PBE /6-311++G(2d,2p) optimised Cartesian coordinates $(\AA)$ of $\mathrm{TS}_{\text {Iint } \rightarrow \text { II }}$. Table S49. QCISD(T)/CBS(MP2)//method calculated energies.

Table S50. QCISD(T)/CBS(QCISD)//QCISD/6-311++G(d,p) calculated energies (Hartrees). 
Table S1. Non-ZPVE corrected relative (to $\left.\mathbf{I}_{\mathrm{f}}\right)$ energies $(\mathrm{kcal} / \mathrm{mol}){ }^{a}$

\begin{tabular}{|c|c|c|c|c|c|c|c|c|c|c|c|}
\hline & QCISD $(\mathrm{T})^{b}$ & MP2 & B3LYP & QCISD $^{c}$ & PBE & PW91 & B3P86 & BLYP & PBE1 & B971 & BHandHLYP \\
\hline $\mathrm{TS}_{\mathbf{I f} \rightarrow \mathbf{I} p}$ & +2.4 & +2.2 & +2.4 & +1.9 & +3.0 & +2.9 & +3.1 & +2.0 & +3.3 & +2.4 & +2.7 \\
\hline $\mathbf{I}_{\mathrm{p}}$ & -3.4 & -5.2 & -1.6 & -2.6 & -1.4 & -1.5 & -1.1 & -2.4 & -0.9 & -2.0 & -0.6 \\
\hline $\mathrm{TS}_{\mathbf{I f} \rightarrow \mathbf{I d}}$ & +3.0 & +2.6 & +3.2 & +3.3 & +3.0 & +2.9 & +3.4 & +2.4 & +3.6 & +2.9 & +4.0 \\
\hline $\mathbf{I}_{\mathrm{d}}$ & -1.1 & -3.5 & +2.3 & +0.3 & +1.5 & +1.5 & +1.7 & +1.9 & +1.2 & +1.2 & +3.0 \\
\hline $\mathrm{TS}_{\mathrm{I} \mathrm{p} \rightarrow \mathbf{I d}}$ & +1.5 & +3.8 & +4.2 & +2.7 & +3.5 & +3.5 & +3.8 & +3.5 & +3.5 & +3.2 & +5.1 \\
\hline $\mathrm{TS}_{\mathrm{I} \mathrm{p} \rightarrow \mathrm{Iint}}$ & +9.0 & +6.5 & +15.2 & +9.5 & +11.3 & +11.9 & +11.6 & +16.4 & +9.9 & +12.7 & +15.2 \\
\hline $\mathrm{TS}_{\mathbf{I d} \rightarrow \mathbf{I i n t}}$ & +5.7 & +2.7 & +12.0 & +6.3 & +8.1 & +8.7 & +8.4 & +13.4 & +6.8 & +9.5 & +12.0 \\
\hline $\mathbf{I}_{\text {int }}$ & --- & --- & +2.4 & --- & --- & --- & --- & +2.1 & --- & +1.5 & +2.8 \\
\hline $\mathbf{I}_{\text {int }}^{\mathrm{d}}$ & --- & -4.2 & --- & --- & --- & --- & --- & --- & --- & --- & --- \\
\hline $\mathbf{I}_{\text {int }}$ & -0.9 & --- & --- & +0.0 & +1.7 & +1.9 & +1.8 & --- & +1.0 & +1.4 & --- \\
\hline $\mathrm{TS}_{\text {IInt } \rightarrow \mathbf{I I}}$ & +5.8 & -0.3 & +5.2 & +5.3 & +5.5 & +5.4 & +5.8 & +4.4 & +5.8 & +4.9 & +6.0 \\
\hline II & -12.2 & -12.8 & -12.4 & -12.3 & -12.2 & -12.1 & -12.4 & -12.2 & -12.6 & -12.5 & -12.4 \\
\hline MAD $^{\mathrm{e}}$ & --- & 2.2 & 2.5 & 0.7 & 1.5 & 1.7 & 1.7 & 2.7 & 1.3 & 1.7 & 2.8 \\
\hline
\end{tabular}

${ }^{a}$ DFT methods: 6-311++G(2d,2p) basis set. ${ }^{b} \mathrm{QCISD}(\mathrm{T}) / \mathrm{CBS} / / \mathrm{QCISD} / 6-311++\mathrm{G}(\mathrm{d}, \mathrm{p}) . \quad{ }^{\mathrm{c}} \mathrm{QCISD} / 6-311++\mathrm{G}(\mathrm{d}, \mathrm{p})$.

${ }^{\mathrm{d}}$ Erroneously predicted structures. ${ }^{\mathrm{e}}$ Mean absolute deviation.

Table S2. ZPVE corrected relative (to $\mathbf{I}_{\mathrm{f}}$ ) energies $(\mathrm{kcal} / \mathrm{mol}){ }^{a}$

\begin{tabular}{|c|c|c|c|c|c|c|c|c|c|c|}
\hline & $\operatorname{QCISD}(\mathrm{T})^{b}$ & MP2 & B3LYP & PBE & PW91 & B3P86 & BLYP & PBE1 & B971 & BHandHLYP \\
\hline $\mathrm{TS}_{\mathbf{I f} \rightarrow \mathbf{I p}}$ & +2.8 & +3.2 & +3.1 & +3.8 & +3.7 & +3.8 & +2.6 & +4.0 & +3.0 & +3.4 \\
\hline $\mathbf{I}_{\mathrm{p}}$ & -1.9 & -3.3 & +0.1 & +0.5 & +0.3 & +0.7 & -0.6 & +0.8 & -0.3 & +0.9 \\
\hline $\mathrm{TS}_{\mathbf{I f} \rightarrow \mathbf{I d}}$ & +3.1 & +3.4 & +3.6 & +3.6 & +3.5 & +3.9 & +3.0 & +4.1 & +3.4 & +4.3 \\
\hline $\mathbf{I}_{\mathrm{d}}$ & +0.3 & -1.9 & +3.6 & +3.0 & +3.0 & +3.1 & +3.2 & +2.8 & +2.7 & +4.3 \\
\hline $\mathrm{TS}_{\mathrm{Ip} \rightarrow \mathbf{I d}}$ & +2.8 & +1.3 & +5.5 & +5.0 & +5.0 & +5.3 & +4.8 & +5.0 & +4.6 & +6.4 \\
\hline $\mathrm{TS}_{\text {Ip } \rightarrow \text { Iint }}$ & +9.6 & +7.0 & +15.6 & +12.0 & +12.6 & +12.3 & +16.8 & +10.7 & +13.1 & +15.6 \\
\hline $\mathrm{TS}_{\mathrm{Id} \rightarrow \mathrm{Iint}}$ & +6.5 & +3.9 & +12.7 & +9.1 & +9.7 & +9.3 & +14.0 & +7.8 & +10.1 & +12.6 \\
\hline $\mathbf{I}_{\text {int }}{ }^{c}$ & --- & --- & +2.6 & --- & --- & --- & +2.4 & --- & +1.9 & +2.8 \\
\hline $\mathbf{I}_{\text {int }}{ }^{c}$ & --- & -3.5 & --- & --- & --- & --- & --- & --- & --- & --- \\
\hline $\mathbf{I}_{\text {int }}$ & -0.2 & --- & --- & +2.5 & +2.7 & +2.5 & --- & +1.9 & +2.0 & --- \\
\hline $\mathrm{TS}_{\text {Iint } \rightarrow \text { II }}$ & +4.7 & +5.1 & +4.8 & +4.9 & +5.2 & +5.1 & +4.1 & +5.1 & +4.6 & +5.1 \\
\hline II & -12.8 & -13.2 & -12.8 & -12.5 & -12.4 & -12.8 & -12.5 & -13.1 & -13.0 & -13.0 \\
\hline
\end{tabular}

${ }^{a}$ DFT methods: 6-311++G(2d,2p) basis set. ${ }^{\mathrm{b}} \mathrm{QCISD}(\mathrm{T}) / \mathrm{CBS} / / \mathrm{QCISD} / 6-311++\mathrm{G}(\mathrm{d}, \mathrm{p}) .{ }^{\mathrm{c}}$ Erroneously predicted structures. 
Table S3. Non-ZPVE corrected 6-311++G(2d,2p) electronic energies (Hartrees).

\begin{tabular}{|c|c|c|c|c|c|c|c|c|c|c|}
\hline & B3LYP & B3P86 & MP2 & PBE & BLYP & BHandHLYP & PW91 & B971 & PBE1 & QCISD $^{a}$ \\
\hline $\mathbf{I}_{\mathrm{f}}$ & -235.030195 & -235.866088 & -234.303945 & -234.692147 & -234.886056 & -234.873089 & -234.911687 & -234.951335 & -234.733503 & -234.320212 \\
\hline $\mathbf{I}_{\mathrm{p}}$ & -235.032665 & -235.867779 & -234.312222 & -234.694307 & -234.889890 & -234.874121 & -234.914092 & -234.954570 & -234.734952 & -234.324397 \\
\hline $\mathbf{I}_{\mathrm{d}}$ & -235.026459 & -235.863446 & -234.309521 & -234.689803 & -234.882997 & -234.868260 & -234.909290 & -234.949361 & -234.731544 & -234.369014 \\
\hline $\mathrm{TS}_{\mathrm{If} \rightarrow \mathrm{Ip}}$ & -235.026304 & -235.861204 & -234.300510 & -234.687343 & -234.882916 & -234.868751 & -234.907082 & -234.947574 & -234.728275 & -234.317201 \\
\hline $\mathrm{TS}_{\mathrm{If} \rightarrow \mathrm{Id}}$ & -235.025126 & -235.860611 & -234.299801 & -234.687343 & -234.882273 & -234.866712 & -234.907031 & -234.946697 & -234.727757 & -234.314989 \\
\hline $\mathrm{TS}_{\mathrm{Ip} \rightarrow \mathrm{Id}}$ & -235.023503 & -235.859995 & -234.305046 & -234.686557 & -234.880430 & -234.864886 & -234.906103 & -234.946259 & -234.727880 & -234.315880 \\
\hline $\mathrm{TS}_{\mathrm{Ip} \rightarrow \mathrm{Iint}}$ & -235.006040 & -235.847555 & -234.293615 & -234.674209 & -234.859949 & -234.848791 & -234.892706 & -234.931055 & -234.717664 & -234.305105 \\
\hline $\mathrm{TS}_{\mathrm{Id} \rightarrow \mathrm{Iint}}$ & -235.011037 & -235.852672 & -234.299713 & -234.679193 & -234.864710 & -234.853991 & -234.897751 & -234.936178 & -234.722627 & -234.310220 \\
\hline & -235.026414 & & & & -234.882701 & -234.868649 & & -234.948873 & & \\
\hline $\mathbf{I}_{\mathrm{int}}{ }^{\mathrm{b}}$ & & & -234.310673 & & & & & & & -234.319301 \\
\hline $\mathbf{I}_{\mathrm{int}}$ & & -235.863243 & & -234.689471 & & & -234.908731 & -234.949165 & -234.731861 & -234.320206 \\
\hline $\mathrm{TS}_{\text {Iint } \rightarrow \text { II }}$ & -235.021909 & -235.856816 & -234.304414 & -234.683453 & -234.879000 & -234.863550 & -234.903026 & -234.943520 & -234.724220 & -234.311841 \\
\hline II & -235.049932 & -235.885903 & -234.324288 & -234.711629 & -234.905433 & -234.892845 & -234.931004 & -234.971305 & -234.753645 & -234.339818 \\
\hline
\end{tabular}

${ }^{\mathrm{a}} 6-311++\mathrm{G}(\mathrm{d}, \mathrm{p})$ basis set. ${ }^{\mathrm{b}}$ Erroneously predicted structures. 
Table S4. ZPVE corrected 6-311++G(2d,2p) electronic energies (Hartrees).

\begin{tabular}{|c|c|c|c|c|c|c|c|c|c|c|}
\hline & B3LYP & B3P86 & MP2 & PBE & BLYP & BHandHLYP & PW91 & B971 & PBE1 & QCISD $^{\mathrm{a}}$ \\
\hline $\mathbf{I}_{\mathrm{f}}$ & -234.875908 & -235.711553 & -234.148421 & -234.542442 & -234.736272 & -234.713130 & -234.761649 & -234.797604 & -234.578602 & -234.163918 \\
\hline $\mathbf{I}_{\mathrm{p}}$ & -234.875682 & -235.710472 & -234.153701 & -234.541642 & -234.737267 & -234.711711 & -234.761111 & -234.798119 & -234.577332 & -234.165845 \\
\hline $\mathbf{I}_{\mathrm{d}}$ & -234.870106 & -235.706573 & -234.151440 & -234.537702 & -234.731229 & -234.706356 & -234.756899 & -234.793333 & -234.574217 & -234.210470 \\
\hline $\mathrm{TS}_{\mathrm{If} \rightarrow \mathrm{Ip}}$ & -234.870938 & -235.705451 & -234.143252 & -234.536371 & -234.732093 & -234.707756 & -234.755811 & -234.792775 & -234.572173 & -234.160264 \\
\hline $\mathrm{TS}_{\mathbf{I f} \rightarrow \mathbf{I d}}$ & -234.870100 & -235.705320 & -234.143051 & -234.536676 & -234.731515 & -234.706233 & -234.756011 & -234.792123 & -234.572140 & -234.158428 \\
\hline $\mathrm{TS}_{\mathrm{Ip} \rightarrow \mathrm{Id}}$ & -234.867147 & -235.703145 & -234.146287 & -234.534474 & -234.728583 & -234.702968 & -234.753722 & -234.790275 & -234.570581 & -234.157479 \\
\hline $\mathrm{TS}_{\mathrm{Ip} \rightarrow \text { Iint }}$ & -234.851043 & -235.691948 & -234.137202 & -234.523307 & -234.709523 & -234.688206 & -234.741547 & -234.776662 & -234.561573 & -234.147898 \\
\hline $\mathrm{TS}_{\mathbf{I d} \rightarrow \operatorname{Iint}}$ & -234.855717 & -235.696680 & -234.142154 & -234.527942 & -234.714027 & -234.693094 & -234.746253 & -234.781474 & -234.566178 & -234.152589 \\
\hline $\mathbf{I}_{\mathrm{int}}{ }^{\mathrm{b}}$ & -234.871779 & & & & -234.732375 & -234.708649 & & -234.794654 & & \\
\hline $\mathbf{I}_{\text {int }}{ }^{\mathrm{b}}$ & & & -234.153955 & & & & & & & -234.161933 \\
\hline $\mathbf{I}_{\text {int }}$ & & -235.707549 & & -234.538420 & & & -234.757416 & -234.794476 & -234.575589 & -234.162838 \\
\hline $\mathrm{TS}_{\text {Iint } \rightarrow \text { II }}$ & -234.868291 & -235.703358 & -234.140283 & -234.534592 & -234.729738 & -234.704981 & -234.753395 & -234.790260 & -234.570404 & -234.157222 \\
\hline II & -234.896348 & -235.732002 & -234.169392 & -234.562329 & -234.756124 & -234.733858 & -234.781383 & -234.818252 & -234.599422 & -234.184511 \\
\hline
\end{tabular}

${ }^{\mathrm{a}} 6-311++\mathrm{G}(\mathrm{d}, \mathrm{p})$ basis set. ${ }^{\mathrm{b}}$ Erroneously predicted structures. 
Table S5. QCISD/6-311++G(d,p) optimised Cartesian coordinates ( $)$ of $\mathbf{I}_{\mathrm{f}}$.

$\begin{array}{rrrr}\mathrm{C} & 1.261902469 & -0.744327705 & -0.247356945 \\ \mathrm{C} & 1.277846145 & 0.761079525 & 0.037779102 \\ \mathrm{C} & -0.001046568 & 1.437525480 & -0.016863960 \\ \mathrm{C} & -1.278995035 & 0.759277241 & 0.037630565 \\ \mathrm{C} & -1.260887868 & -0.746104762 & -0.247514173 \\ \mathrm{C} & 0.000933407 & -1.400654062 & 0.323404260 \\ \mathrm{H} & -0.001814070 & 2.525613335 & 0.010332758 \\ \mathrm{H} & 1.525696437 & 0.964211542 & 1.107449863 \\ \mathrm{H} & 2.040518868 & 1.343014248 & -0.485530220 \\ \mathrm{H} & 1.302856683 & -0.891608286 & -1.326850343 \\ \mathrm{H} & 2.161591528 & -1.190977221 & 0.167628589 \\ \mathrm{H} & -1.527297570 & 0.962059674 & 1.107257938 \\ \mathrm{H} & -2.042406874 & 1.340135209 & -0.485799620 \\ \mathrm{H} & -2.159998373 & -1.194028345 & 0.167351899 \\ \mathrm{H} & -1.301494034 & -0.893436301 & -1.327013771 \\ \mathrm{H} & 0.000808880 & -1.319928561 & 1.413527802 \\ \mathrm{H} & 0.001695974 & -2.462100941 & 0.089066225\end{array}$

Table S6. QCISD/6-311++G(d,p) optimised Cartesian coordinates ( $)$ of $\mathbf{I}_{\mathrm{p}}$.

$\begin{array}{rrrr}\text { C } & -1.250901565 & 0.683809914 & -0.241212317 \\ \mathrm{C} & -1.253697939 & -0.855560948 & 0.265301161 \\ \mathrm{C} & -0.000776711 & -1.268354590 & -0.305561481 \\ \mathrm{C} & 1.252656470 & -0.856955069 & 0.264982726 \\ \mathrm{C} & 1.251596736 & 0.682388931 & -0.241222456 \\ \mathrm{C} & 0.000717900 & 1.391308552 & 0.256546710 \\ \mathrm{H} & -0.001140657 & -1.667238415 & -1.317309004 \\ \mathrm{H} & -1.253739601 & -0.839379390 & 1.350877971 \\ \mathrm{H} & -2.122348362 & -1.355365281 & -0.148723863 \\ \mathrm{H} & -1.315405986 & 0.707605447 & -1.328226151 \\ \mathrm{H} & -2.173940597 & 1.094848326 & 0.160335651 \\ \mathrm{H} & 1.253232558 & -0.840992552 & 1.350601930 \\ \mathrm{H} & 2.120667798 & -1.357599635 & -0.149416986 \\ \mathrm{H} & 2.175023836 & 1.092568557 & 0.160370224 \\ \mathrm{H} & 1.316174805 & 0.706153922 & -1.328249777 \\ \mathrm{H} & 0.000766948 & 1.442334606 & 1.345421305 \\ \mathrm{H} & 0.001348367 & 2.416549515 & -0.115310642\end{array}$

Table S7. QCISD/6-311++G(d,p) optimised Cartesian coordinates ( $)$ of $\mathbf{I}_{\mathrm{d}}$.

$\begin{array}{rrrr}\text { C } & 0.769898557 & -1.022156874 & -0.228125454 \\ \text { C } & -1.401251440 & -0.027607795 & 0.391359833 \\ \text { C } & 0.754357243 & 0.984600820 & -0.434002622 \\ \text { C } & -0.711729446 & 1.267252269 & -0.102539522 \\ \text { C } & 1.377127457 & -0.128205771 & 0.668539584 \\ \mathrm{C} & -0.698281026 & -1.233232030 & -0.252257648 \\ \mathrm{H} & -2.458500090 & -0.035635124 & 0.145708859 \\ \mathrm{H} & 0.901454577 & 0.701958317 & -1.479697089 \\ \mathrm{H} & -0.777018843 & 2.041485823 & 0.657753453 \\ \mathrm{H} & 0.883648147 & 0.098387997 & 1.603361312 \\ \mathrm{H} & -0.891355106 & -2.155274257 & 0.308549788 \\ \mathrm{H} & 1.390710767 & -1.481858246 & -0.990003301 \\ \mathrm{H} & -1.332690955 & -0.096553263 & 1.474981887\end{array}$




$\begin{array}{rrrr}\mathrm{H} & 1.465151076 & 1.788969638 & -0.254282215 \\ \mathrm{H} & -1.200516322 & 1.643126049 & -0.998289130 \\ \mathrm{H} & 2.456382985 & -0.094257471 & 0.692936142 \\ \mathrm{H} & -1.020863533 & -1.420389158 & -1.275424870\end{array}$

Table S8. QCISD/6-311++G(d,p) optimised Cartesian coordinates ( $\mathrm{A})$ of $\mathbf{I}_{\text {int }}$.

$\begin{array}{lrrr}\mathrm{C} & 0.729863270 & -0.480158724 & -0.630570631 \\ \mathrm{C} & -1.367147102 & -0.230400196 & 0.530341970 \\ \mathrm{C} & 0.423349956 & 0.894394584 & -0.592666881 \\ \mathrm{C} & -0.937420505 & 1.137115241 & -0.044057179 \\ \mathrm{C} & 1.602993355 & -0.026676163 & 0.726307390 \\ \mathrm{C} & -0.531485375 & -1.256784991 & -0.265851983 \\ \mathrm{H} & -2.434251521 & -0.397983277 & 0.434446150 \\ \mathrm{H} & 1.080137343 & 1.668128050 & -0.971790968 \\ \mathrm{H} & -1.008392572 & 1.988144525 & 0.628070266 \\ \mathrm{H} & 1.358547413 & -0.825257441 & 1.418470293 \\ \mathrm{H} & -0.318870376 & -2.167821194 & 0.286431668 \\ \mathrm{H} & 1.447541423 & -0.874074868 & -1.335335695 \\ \mathrm{H} & -1.124084304 & -0.273374146 & 1.590677263 \\ \mathrm{H} & 1.358834517 & 0.912704218 & 1.232482760 \\ \mathrm{H} & -1.520695694 & 1.391990879 & -0.940487724 \\ \mathrm{H} & 2.642686886 & 0.000400996 & 0.429773716 \\ \mathrm{H} & -1.031135748 & -1.537384485 & -1.192907536\end{array}$

Table S9. QCISD/6-311++G(d,p) optimised Cartesian coordinates ( $)$ of II.

$\begin{array}{lrrr}\mathrm{C} & 1.508510543 & 0.724558048 & -0.210518508 \\ \mathrm{C} & 0.076010800 & 1.198763327 & 0.104082314 \\ \mathrm{C} & -0.774640415 & 0.009415928 & -0.026121179 \\ \mathrm{C} & -2.238618927 & 0.006087792 & 0.006182181 \\ \mathrm{C} & 0.060203930 & -1.188273097 & -0.144454067 \\ \mathrm{C} & 1.484000362 & -0.745868858 & 0.242095670 \\ \mathrm{H} & 0.018697747 & -1.344856601 & -1.242814283 \\ \mathrm{H} & -0.009935207 & 1.406305118 & 1.189323837 \\ \mathrm{H} & -0.311189440 & 2.083953672 & -0.396605513 \\ \mathrm{H} & 1.686931178 & 0.793631413 & -1.283345306 \\ \mathrm{H} & 2.260188465 & 1.319975858 & 0.296032611 \\ \mathrm{H} & -2.674331358 & 0.947849169 & -0.313370692 \\ \mathrm{H} & -2.658348142 & -0.846102784 & -0.523592978 \\ \mathrm{H} & -2.491817054 & -0.136034832 & 1.068431762 \\ \mathrm{H} & -0.363832589 & -2.087500667 & 0.298322163 \\ \mathrm{H} & 1.607456224 & -0.819967684 & 1.322401590 \\ \mathrm{H} & 2.247353935 & -1.355805799 & -0.228519617\end{array}$

Table S10. QCISD/6-311++G(d,p) optimised Cartesian coordinates ( $\AA$ ) of $\mathrm{TS}_{\mathbf{I f} \rightarrow \mathbf{I} \mathrm{p}}$.

$\begin{array}{rrrr}\text { C } & 1.266221 & -0.717857 & -0.242442 \\ \text { C } & 1.273611 & 0.799120 & 0.157317 \\ \text { C } & -0.003143 & 1.439098 & -0.141256 \\ \text { C } & -1.277162 & 0.793491 & 0.156876 \\ \text { C } & -1.262947 & -0.723446 & -0.242846 \\ \text { C } & 0.003091 & -1.415681 & 0.265155 \\ \text { H } & -0.005254 & 2.431587 & -0.605599 \\ \text { H } & 1.339515 & 0.834535 & 1.264806\end{array}$




$\begin{array}{rrrr}\mathrm{H} & 2.119255 & 1.358324 & -0.248400 \\ \mathrm{H} & 1.333845 & -0.783777 & -1.335082 \\ \mathrm{H} & 2.173764 & -1.166371 & 0.170968 \\ \mathrm{H} & -1.343601 & 0.828643 & 1.264343 \\ \mathrm{H} & -2.125125 & 1.348952 & -0.249142 \\ \mathrm{H} & -2.168631 & -1.175957 & 0.170286 \\ \mathrm{H} & -1.329938 & -0.789680 & -1.335507 \\ \mathrm{H} & 0.002978 & -1.443576 & 1.363409 \\ \mathrm{H} & 0.005443 & -2.455139 & -0.083046\end{array}$

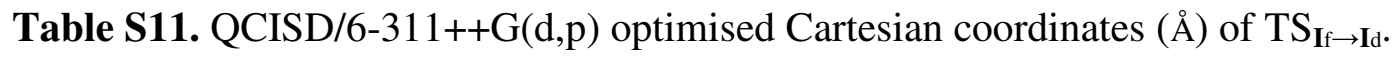

$\begin{array}{rrrr}\text { C } & -0.109554 & -1.385577 & -0.270671 \\ \mathrm{C} & -1.109499 & 0.785381 & 0.433454 \\ \mathrm{C} & 1.374761 & 0.552568 & -0.159153 \\ \mathrm{C} & 0.100494 & 1.417791 & -0.285297 \\ \mathrm{C} & 1.083109 & -0.924440 & 0.399191 \\ \mathrm{C} & -1.338972 & -0.609026 & -0.158458 \\ \mathrm{H} & -2.008530 & 1.392135 & 0.297996 \\ \mathrm{H} & 1.888034 & 0.445911 & -1.118247 \\ \mathrm{H} & 0.291715 & 2.416572 & 0.117080 \\ \mathrm{H} & 0.828825 & -0.767674 & 1.460160 \\ \mathrm{H} & -1.983648 & -1.253153 & 0.483843 \\ \mathrm{H} & -0.102804 & -2.284645 & -0.895154 \\ \mathrm{H} & -0.925815 & 0.720403 & 1.513183 \\ \mathrm{H} & 2.085479 & 0.970453 & 0.558667 \\ \mathrm{H} & -0.156464 & 1.540714 & -1.344823 \\ \mathrm{H} & 1.944461 & -1.584223 & 0.300088 \\ \mathrm{H} & -1.863290 & -0.616675 & -1.127186\end{array}$

Table S12. QCISD/6-311++G(d,p) optimised Cartesian coordinates ( $)$ of $\mathrm{TS}_{I_{\mathrm{p} \rightarrow I d}}$.

\begin{tabular}{|c|c|c|c|}
\hline$C$ & -1.514043 & -0.026933 & 0.162593 \\
\hline & -0.646637 & -1.317432 & -0.072826 \\
\hline & 0.798283 & -1.004132 & -0.235480 \\
\hline & 1.462738 & -0.066910 & 0.581492 \\
\hline 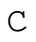 & 0.710824 & 0.983747 & -0.482438 \\
\hline & -0.679187 & 1.278026 & 0.064967 \\
\hline & 1.360077 & -1.452733 & -1.056613 \\
\hline & -0.707891 & -1.970170 & 0.812140 \\
\hline 1 & -1.002440 & -1.891798 & -0.930915 \\
\hline$\vec{H}$ & -2.302581 & -0.027429 & -0.594534 \\
\hline H & -2.007476 & -0.087887 & 1.135160 \\
\hline H & 1.049115 & 0.156215 & 1.562950 \\
\hline 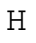 & 2.544932 & -0.000414 & 0.503240 \\
\hline $\mathrm{H}$ & 1.439269 & 1.801282 & -0.484329 \\
\hline 1 & 0.665094 & 0.639158 & -1.528928 \\
\hline & -0.584993 & 1.758895 & 1.043604 \\
\hline & -1.171578 & 1.991994 & $-0.60353^{7}$ \\
\hline
\end{tabular}

Table S13. QCISD/6-311++G(d,p) optimised Cartesian coordinates $(\AA)$ of $\mathrm{TS}_{\mathrm{I}_{\mathrm{p}} \rightarrow \mathrm{Int}}$.

$\begin{array}{rrrr}\text { C } & -0.56128200 & 0.84215700 & -0.46446500 \\ \text { C } & -1.71567200 & -0.11644600 & 0.46619000 \\ \text { C } & -0.70508200 & -0.68070300 & -0.46318700 \\ \text { C } & 0.59913700 & -1.25399800 & 0.12200400 \\ \text { C } & 1.61757100 & -0.09927600 & -0.00331800\end{array}$




$\begin{array}{rrrr}\mathrm{C} & 0.78064600 & 1.18080900 & 0.20870800 \\ \mathrm{H} & -1.11405800 & -1.11754900 & -1.36952700 \\ \mathrm{H} & -1.51060500 & -0.14757000 & 1.53620200 \\ \mathrm{H} & -2.75522500 & -0.05309800 & 0.14768900 \\ \mathrm{H} & -0.96095300 & 1.37857700 & -1.32423500 \\ \mathrm{H} & -1.43584200 & 1.19789900 & 0.34578700 \\ \mathrm{H} & 0.45192400 & -1.54182300 & 1.16969900 \\ \mathrm{H} & 0.90456200 & -2.14780700 & -0.42694600 \\ \mathrm{H} & 2.44110500 & -0.17784600 & 0.71134300 \\ \mathrm{H} & 2.04510800 & -0.09122100 & -1.01294800 \\ \mathrm{H} & 0.63467800 & 1.38756100 & 1.27567300 \\ \mathrm{H} & 1.20740300 & 2.07762600 & -0.24833000\end{array}$

Table S14. QCISD/6-311++G(d,p) optimised Cartesian coordinates ( $)$ of $\mathrm{TS}_{\mathbf{I d} \rightarrow \mathbf{I i n t}}$.

$\begin{array}{rrrr}\text { C } & 0.78611000 & -0.63166600 & -0.49029400 \\ \text { C } & -1.39988400 & -0.17085800 & 0.47983400 \\ \text { C } & 0.59776700 & 0.91514500 & -0.41037900 \\ \text { C } & -0.89017600 & 1.15852900 & -0.11040300 \\ \text { C } & 1.53257900 & -0.14386200 & 0.66769600 \\ \text { C } & -0.59243500 & -1.25670200 & -0.25885600 \\ \text { H } & -2.47741800 & -0.28852900 & 0.34266200 \\ \text { H } & 1.15869700 & 1.47399900 & -1.15851600 \\ \text { H } & -1.06249300 & 2.02866000 & 0.52796000 \\ \text { H } & 1.10960900 & -0.22771000 & 1.66730800 \\ \text { H } & -0.52227500 & -2.19887900 & 0.29288400 \\ \text { H } & 1.39172700 & -0.96930400 & -1.32655000 \\ \text { H } & -1.21044600 & -0.20755600 & 1.55968000 \\ \text { H } & 1.18888600 & 1.26766800 & 0.56450000 \\ \text { H } & -1.35204200 & 1.35288500 & -1.08619800 \\ \text { H } & 2.60628900 & 0.01584700 & 0.58865400 \\ \text { H } & -1.03430000 & -1.47059900 & -1.23798000\end{array}$

Table S15. QCISD/6-311++G(d,p) optimised Cartesian coordinates $(\AA)$ of $\mathrm{TS}_{\text {Iint } \rightarrow \text { II }}$.

$\begin{array}{rrrr}\mathrm{C} & -1.49874626 & -0.72076220 & 0.26642131 \\ \mathrm{C} & -0.10760353 & -1.21994675 & -0.22383807 \\ \mathrm{C} & 0.78427784 & 0.04248708 & -0.33807905 \\ \mathrm{C} & 2.19487199 & 0.02386591 & 0.29348993 \\ \mathrm{C} & -0.07319929 & 1.17388571 & -0.06977577 \\ \mathrm{C} & -1.48400020 & 0.81226823 & 0.05415694 \\ \mathrm{H} & 0.94601877 & 0.32077960 & -1.42179903 \\ \mathrm{H} & 0.33524372 & -1.93052164 & 0.47964954 \\ \mathrm{H} & -0.17869890 & -1.72124889 & -1.19361170 \\ \mathrm{H} & -2.32291351 & -1.19371017 & -0.27118993 \\ \mathrm{H} & -1.63264130 & -0.93273784 & 1.33162768 \\ \mathrm{H} & 2.77729011 & -0.77608773 & -0.16977738 \\ \mathrm{H} & 2.71554419 & 0.97487732 & 0.14524755 \\ \mathrm{H} & 2.10730330 & -0.18090468 & 1.36508768 \\ \mathrm{H} & 0.29386337 & 2.20454250 & -0.05300487 \\ \mathrm{H} & -1.99632396 & 1.43289595 & 0.80315117 \\ \mathrm{H} & -1.93828907 & 1.13132764 & -0.90963246\end{array}$

Table S16. B3LYP/6-311++G(2d,2p) optimised Cartesian coordinates ( $)$ of $\mathbf{I}_{\mathrm{f}}$.
C -0.22184500
1.42495700
0.01985400
C 1.36736000
$-0.53504600$
$-0.22234500$ 


$\begin{array}{lrrr}\mathrm{C} & -1.13489800 & -0.92537400 & -0.23138900 \\ \mathrm{C} & 0.21559100 & -1.38001800 & 0.33257200 \\ \mathrm{C} & -1.37596200 & 0.56581100 & 0.01976000 \\ \mathrm{C} & 1.13931200 & 0.95863800 & 0.02619100 \\ \mathrm{H} & 1.46546900 & -0.69508600 & -1.29749800 \\ \mathrm{H} & -1.17100600 & -1.10423000 & -1.30747700 \\ \mathrm{H} & 0.20186300 & -1.31787900 & 1.42458500 \\ \mathrm{H} & -1.77755500 & 0.76037900 & 1.04403400 \\ \mathrm{H} & 1.45708600 & 1.26775600 & 1.05171600 \\ \mathrm{H} & -0.38929900 & 2.49833300 & 0.09171600 \\ \mathrm{H} & 2.31695800 & -0.82875400 & 0.22038600 \\ \mathrm{H} & -1.95266700 & -1.49678200 & 0.20314800 \\ \mathrm{H} & 0.37974100 & -2.42792100 & 0.08934800 \\ \mathrm{H} & -2.17482800 & 1.02663800 & -0.57918200 \\ \mathrm{H} & 1.76197100 & 1.64111100 & -0.57035100\end{array}$

Table S17. B3LYP/6-311++G(2d,2p) optimised Cartesian coordinates ( $\AA$ ) of $\mathbf{I}_{\mathrm{p}}$.

$\begin{array}{lrrr}\text { C } & 0.00135700 & -1.28358300 & -0.29314000 \\ \text { C } & -1.25612800 & 0.69039200 & -0.23850000 \\ \text { C } & 1.25457100 & 0.69265700 & -0.23876200 \\ \text { C } & -0.00135300 & 1.39938500 & 0.24056400 \\ \text { C } & 1.24970100 & -0.86545000 & 0.26519600 \\ \text { C } & -1.24793700 & -0.86765100 & 0.26495700 \\ \text { H } & -1.34715000 & 0.71275500 & -1.32329600 \\ \mathrm{H} & 1.34527000 & 0.71494800 & -1.32358400 \\ \mathrm{H} & -0.00129700 & 1.48630800 & 1.32790200 \\ \mathrm{H} & 1.26566100 & -0.84085200 & 1.35175200 \\ \mathrm{H} & -1.26405900 & -0.84348800 & 1.35153200 \\ \mathrm{H} & 0.00186700 & -1.71057700 & -1.29374000 \\ \mathrm{H} & -2.17619700 & 1.08998600 & 0.18443500 \\ \mathrm{H} & 2.17405900 & 1.09393900 & 0.18384900 \\ \mathrm{H} & -0.00228700 & 2.41853700 & -0.15603300 \\ \mathrm{H} & 2.12348600 & -1.35600700 & -0.15215700 \\ \mathrm{H} & -2.12062500 & -1.36004700 & -0.15254700\end{array}$

Table S18. B3LYP/6-311++G(2d,2p) optimised Cartesian coordinates ( $\AA$ ) of $\mathbf{I}_{\mathrm{d}}$.

$\begin{array}{lrrr}\text { C } & 0.76596500 & -1.06319500 & -0.22756000 \\ \mathrm{C} & -1.40219700 & -0.03727900 & 0.38088700 \\ \mathrm{C} & 0.75993400 & 1.04818300 & -0.40293400 \\ \mathrm{C} & -0.72483000 & 1.26602300 & -0.11582000 \\ \mathrm{C} & 1.38884700 & -0.12588500 & 0.61737600 \\ \mathrm{C} & -0.68784800 & -1.25093100 & -0.23912600 \\ \mathrm{H} & -2.45799600 & -0.05997200 & 0.12420000 \\ \mathrm{H} & 0.96406900 & 0.83267800 & -1.45319500 \\ \mathrm{H} & -0.84790600 & 2.04664400 & 0.63271100 \\ \mathrm{H} & 0.94268900 & 0.07656900 & 1.58392400 \\ \mathrm{H} & -0.88442100 & -2.16037300 & 0.35283900 \\ \mathrm{H} & 1.36918700 & -1.55522000 & -0.98592800 \\ \mathrm{H} & -1.35029400 & -0.09172700 & 1.46710100 \\ \mathrm{H} & 1.42361800 & 1.87177200 & -0.13795400 \\ \mathrm{H} & -1.20538200 & 1.61491900 & -1.02837700 \\ \mathrm{H} & 2.47056500 & -0.10269400 & 0.61668900 \\ \mathrm{H} & -1.02335100 & -1.49409100 & -1.24894500\end{array}$


Table S19. B3LYP/6-311++G(2d,2p) optimised Cartesian coordinates ( $\AA$ ) of $\mathbf{I}_{\text {int }}$.

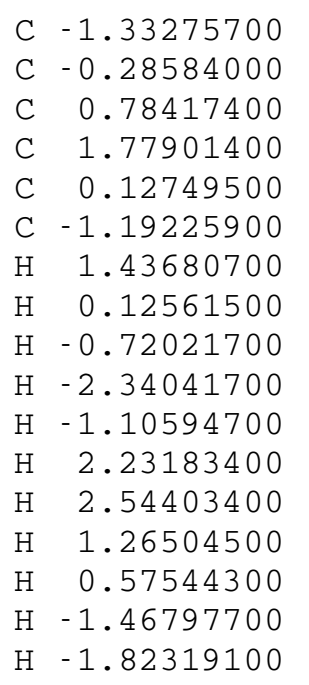

Table S20. B3LYP/6-311++G(2d,2p) optimised Cartesian coordinates ( $\AA$ ) of II.

$\begin{array}{lr}\mathrm{H} & -0.31081600 \\ \mathrm{C} & 0.07026600 \\ \mathrm{C} & -0.77661000 \\ \mathrm{C} & 0.05568600 \\ \mathrm{C} & 1.48801300 \\ \mathrm{C} & 1.51340900 \\ \mathrm{H} & -0.03901400 \\ \mathrm{H} & 2.24216700 \\ \mathrm{H} & 1.63613500 \\ \mathrm{H} & 1.72766500 \\ \mathrm{H} & 2.24923100 \\ \mathrm{C} & -2.23408300 \\ \mathrm{H} & -2.66125700 \\ \mathrm{H} & -2.67809400 \\ \mathrm{H} & -2.50408900 \\ \mathrm{H} & -0.36347800 \\ \mathrm{H} & 0.00147100\end{array}$

0.50009600
-0.39810300
-0.63221200
0.64123500
-0.45026300
0.13279900
-1.49611700
0.04464500
-1.35944800
0.33795400
1.55700200
0.72510500
0.44067600
1.57218500
-0.70133200
0.86985800
-0.75183400

$\begin{array}{rr}-0.54879200 & 0.50009600 \\ -1.24576900 & -0.39810300 \\ -0.16502800 & -0.63221200 \\ 0.02015000 & 0.64123500 \\ 1.08817600 & -0.45026300 \\ 0.94371400 & 0.13279900 \\ -0.26078000 & -1.49611700 \\ -2.14909200 & 0.04464500 \\ -1.52151300 & -1.35944800 \\ -0.91875300 & 0.33795400 \\ -0.68281500 & 1.55700200 \\ -0.96726200 & 0.72510500 \\ 0.76298300 & 0.44067600 \\ 0.24457900 & 1.57218500 \\ 2.04520400 & -0.70133200 \\ 1.69958900 & 0.86985800 \\ 1.19315400 & -0.75183400\end{array}$

$\begin{array}{rr}2.06557100 & -0.45976100 \\ 1.19773100 & 0.08108000 \\ 0.00880200 & -0.02580700 \\ -1.18725200 & -0.12972100 \\ -0.74911400 & 0.23378200 \\ 0.72895900 & -0.19522900 \\ 1.47180700 & 1.15096400 \\ -1.35432000 & -0.26012400 \\ -0.84695200 & 1.30972100 \\ 0.82154100 & -1.26038200 \\ 1.32014500 & 0.34136600 \\ 0.00696100 & 0.00680600 \\ -0.83813100 & -0.53301300 \\ 0.95253300 & -0.29448600 \\ -0.15760500 & 1.06426500 \\ -2.07966500 & 0.33725400 \\ -1.39144500 & -1.22127600\end{array}$

Table S21. B3LYP/6-311++G(2d,2p) optimised Cartesian coordinates ( $\AA$ ) of $\mathrm{TS}_{\mathrm{If} \rightarrow \mathbf{I} \text {. }}$.

C 1.26486200

C 1.26599500

C 0.00008900

C -1.26589500

C -1.26495500

C -0.00008400

H 0.00014900

H 1.36118400

$\mathrm{H} \quad 2.10830700$

$\mathrm{H} \quad 1.35844400$

H 2.16214700

$\mathrm{H}-1.36109700$

$\mathrm{H}-2.10813400$

$\mathrm{H}-2.16228500$
$-0.72524900$
$-0.23527300$
0.80495700
0.14978200
$-0.13707600$
0.14977000
0.80510800
$-0.72510400$
$-1.42139600$
$-0.23525900$
0.25120200
$-0.61256400$
0.83414400
1. 25074200
1. 35523000
$-0.79071300$
$-1.16457400$
0.83433100
1.35547800
$-1.16431900$
$-1.31933800$
0.19381700
1. 25072800
$-0.26046200$
0.19385100 


$\begin{array}{lllr}\mathrm{H}-1.35856100 & -0.79057200 & -1.31932100 \\ \mathrm{H}-0.00008100 & -1.48586000 & 1.34154100 \\ \mathrm{H}-0.00014400 & -2.44913200 & -0.11743000\end{array}$

Table S22. B3LYP/6-311++G(2d,2p) optimised Cartesian coordinates ( $)$ of $\mathrm{TS}_{\mathbf{I} \mathbf{f} \rightarrow \mathbf{I d} \text {. }}$

$\begin{array}{lrrr}\text { C } & 0.13564800 & -1.39207300 & -0.23408700 \\ \text { C } & -1.24007900 & 0.57746000 & 0.41208000 \\ \text { C } & 1.24739400 & 0.75868200 & -0.21661600 \\ \text { C } & -0.13154900 & 1.44210900 & -0.22823100 \\ \text { C } & 1.21363300 & -0.73426600 & 0.42656000 \\ \text { C } & -1.19374600 & -0.82499500 & -0.18788400 \\ \text { H } & -2.21991800 & 1.01872100 & 0.24612200 \\ \text { H } & 1.67353900 & 0.67316200 & -1.21199500 \\ \text { H } & -0.07816800 & 2.39394200 & 0.29637100 \\ \mathrm{H} & 0.94752300 & -0.54227900 & 1.47221300 \\ \mathrm{H} & -1.79588000 & -1.55597400 & 0.39146300 \\ \mathrm{H} & 0.30776800 & -2.27653200 & -0.84412900 \\ \mathrm{H} & -1.10281400 & 0.52992500 & 1.49366600 \\ \mathrm{H} & 1.96856000 & 1.27635900 & 0.41135900 \\ \mathrm{H} & -0.41423200 & 1.66443300 & -1.25702800 \\ \mathrm{H} & 2.17473500 & -1.23004900 & 0.35604900 \\ \mathrm{H} & -1.64892600 & -0.91320900 & -1.18502300\end{array}$

Table S23. B3LYP/6-311++G(2d,2p) optimised Cartesian coordinates ( $)$ of $\mathrm{TS}_{\mathbf{I}_{\mathrm{p} \rightarrow \mathbf{I d}} \text {. }}$.

$\begin{array}{lrrr}\text { C } & -1.50413200 & 0.05788000 & 0.15932100 \\ \mathrm{C} & -0.72108100 & -1.28526700 & -0.09228900 \\ \mathrm{C} & 0.72447600 & -1.09688900 & -0.21112300 \\ \mathrm{C} & 1.43841500 & -0.15862700 & 0.55821900 \\ \mathrm{C} & 0.78505900 & 0.99896200 & -0.45273500 \\ \mathrm{C} & -0.60889400 & 1.31912300 & 0.04946200 \\ \mathrm{H} & 1.26010500 & -1.61170500 & -1.00432200 \\ \mathrm{H} & -0.85956900 & -1.93482800 & 0.78520300 \\ \mathrm{H} & -1.10890500 & -1.83266200 & -0.94701500 \\ \mathrm{H} & -2.30335200 & 0.08913500 & -0.57694000 \\ \mathrm{H} & -1.98436800 & 0.02638500 & 1.13253700 \\ \mathrm{H} & 1.06882600 & 0.08432000 & 1.54772200 \\ \mathrm{H} & 2.51770500 & -0.18085900 & 0.48160800 \\ \mathrm{H} & 1.52842200 & 1.79056500 & -0.35552600 \\ \mathrm{H} & 0.79587700 & 0.71126500 & -1.50780600 \\ \mathrm{H} & -0.53242700 & 1.81426100 & 1.01600200 \\ \mathrm{H} & -1.06536900 & 2.03303300 & -0.63658900\end{array}$

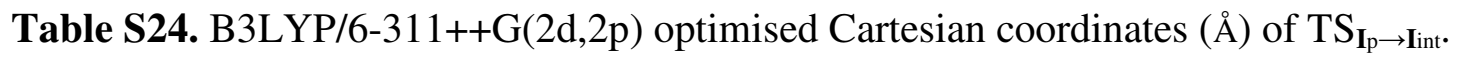

$\begin{array}{lr}\text { C } & -0.57751400 \\ \text { C } & -1.71559700 \\ \text { C } & -0.70508900 \\ \text { C } & 0.60888100 \\ \text { C } & 1.61325200 \\ \text { C } & 0.76531700 \\ \text { H } & -1.11841400 \\ \text { H } & -1.50752800 \\ \text { H } & -2.75381400 \\ \text { H } & -1.01541600\end{array}$

0.83010600

$-0.14522800$

$-0.68468300$

$-1.25008400$

$-0.08103100$

1.19934200

$-1.13054100$

$-0.20011200$

$-0.10731900$

1.36954200 


$\begin{array}{rrrr}\mathrm{H} & -1.45954500 & 1.15565400 & 0.38926700 \\ \mathrm{H} & 0.47046100 & -1.58735700 & 1.13519600 \\ \mathrm{H} & 0.93114900 & -2.11225100 & -0.46927600 \\ \mathrm{H} & 2.39527100 & -0.14492700 & 0.77823600 \\ \mathrm{H} & 2.09957100 & -0.07884500 & -0.94933100 \\ \mathrm{H} & 0.64171900 & 1.47857700 & 1.22594600 \\ \mathrm{H} & 1.17919200 & 2.06649000 & -0.33116400\end{array}$

Table S25. B3LYP/6-311++G(2d,2p) optimised Cartesian coordinates (Å) of $\mathrm{TS}_{\mathbf{I d} \rightarrow \mathbf{I n t}}$.

$\begin{array}{rrrr}\mathrm{C} & 0.75033200 & -0.66305700 & -0.49417500 \\ \mathrm{C} & -1.41174200 & -0.09802300 & 0.47581700 \\ \mathrm{C} & 0.60984400 & 0.87301100 & -0.48022500 \\ \mathrm{C} & -0.84570200 & 1.19394400 & -0.14062400 \\ \mathrm{C} & 1.54368300 & -0.15572900 & 0.63178400 \\ \mathrm{C} & -0.63728500 & -1.23780800 & -0.21201100 \\ \mathrm{H} & -2.48511800 & -0.17859400 & 0.32602300 \\ \mathrm{H} & 1.18208100 & 1.40837900 & -1.22952600 \\ \mathrm{H} & -0.96348900 & 2.07658800 & 0.48306500 \\ \mathrm{H} & 1.15042500 & -0.21073800 & 1.63951100 \\ \mathrm{H} & -0.59024000 & -2.14745600 & 0.38330100 \\ \mathrm{H} & 1.32558700 & -1.07123400 & -1.31310400 \\ \mathrm{H} & -1.25022700 & -0.10777700 & 1.55428800 \\ \mathrm{H} & 1.25854000 & 1.21465200 & 0.49373600 \\ \mathrm{H} & -1.31635800 & 1.41008000 & -1.10220700 \\ \mathrm{H} & 2.61649800 & -0.05951700 & 0.52203700 \\ \mathrm{H} & -1.09434000 & -1.49781300 & -1.16600700\end{array}$

Table S26. B3LYP/6-311++G(2d,2p) optimised Cartesian coordinates ( $)$ of $\mathrm{TS}_{\text {Int } \rightarrow \text { II }}$.

$\begin{array}{rrrr}\mathrm{C} & 0.78017300 & -0.09836600 & -0.51153600 \\ \mathrm{C} & -1.53968500 & -0.60597700 & 0.22879300 \\ \mathrm{C} & 0.02645800 & 1.11306500 & -0.35546200 \\ \mathrm{C} & -1.28925500 & 0.91664500 & 0.19497300 \\ \mathrm{C} & 2.03724700 & 0.03020300 & 0.45688000 \\ \mathrm{C} & -0.18923500 & -1.26179400 & -0.19004200 \\ \mathrm{H} & -2.33600100 & -0.86459200 & -0.46437000 \\ \mathrm{H} & 0.43351400 & 2.09450300 & -0.58696700 \\ \mathrm{H} & -1.28230700 & 1.41394100 & 1.18648400 \\ \mathrm{H} & 2.59915200 & -0.89232100 & 0.33041900 \\ \mathrm{H} & 0.21628100 & -1.88203200 & 0.60564900 \\ \mathrm{H} & 1.22803200 & -0.12625100 & -1.51406800 \\ \mathrm{H} & -1.86081900 & -0.92771000 & 1.21524400 \\ \mathrm{H} & 1.71864400 & 0.11429700 & 1.49283100 \\ \mathrm{H} & -2.02516600 & 1.53640700 & -0.33911200 \\ \mathrm{H} & 2.66844300 & 0.87412500 & 0.19093800 \\ \mathrm{H} & -0.31399800 & -1.90302000 & -1.05868400\end{array}$

Table S27. MP2/6-311++G(2d,2p) optimised Cartesian coordinates ( $)$ of $\mathbf{I}_{\mathrm{f}}$.

$\begin{array}{rrrr}\text { C } & 1.25705700 & -0.73711600 & -0.23660100 \\ \text { C } & 1.26864000 & 0.76607500 & 0.02977300 \\ \text { C } & -0.00006700 & 1.43987300 & -0.02218100 \\ \text { C } & -1.26871700 & 0.76595500 & 0.02978200 \\ \text { C } & -1.25698200 & -0.73723300 & -0.23660500 \\ \text { C } & 0.00006700 & -1.38310300 & 0.34381300\end{array}$




$\begin{array}{lrrr}\mathrm{H} & -0.00011700 & 2.52732200 & 0.00873700 \\ \mathrm{H} & 1.50515100 & 0.98363600 & 1.10208700 \\ \mathrm{H} & 2.03953000 & 1.35064800 & -0.47784700 \\ \mathrm{H} & 1.29550000 & -0.89712300 & -1.31300500 \\ \mathrm{H} & 2.15717000 & -1.17781600 & 0.18122700 \\ \mathrm{H} & -1.50532100 & 0.98350000 & 1.10207100 \\ \mathrm{H} & -2.03963100 & 1.35045200 & -0.47789500 \\ \mathrm{H} & -2.15705400 & -1.17802700 & 0.18121100 \\ \mathrm{H} & -1.29539900 & -0.89723800 & -1.31301000 \\ \mathrm{H} & 0.00006100 & -1.28473300 & 1.43127800 \\ \mathrm{H} & 0.00011800 & -2.44732100 & 0.12726000\end{array}$

Table S28. MP2/6-311++G(2d,2p) optimised Cartesian coordinates ( $\AA$ ) of $\mathbf{I}_{\mathrm{p}}$.

$\begin{array}{lr}\text { C } & -0.84230300 \\ \text { C } & -1.53613200 \\ \text { C } & -0.52862300 \\ \text { C } & 0.81732600 \\ \text { C } & 1.47563700 \\ \text { C } & 0.53521500 \\ \text { H } & -0.70632000 \\ \mathrm{H} & -1.46777200 \\ \mathrm{H} & -2.53866900 \\ \mathrm{H} & -0.85345600 \\ \mathrm{H} & -1.61383100 \\ \mathrm{H} & 0.81850600 \\ \mathrm{H} & 1.38281400 \\ \mathrm{H} & 2.45243400 \\ \mathrm{H} & 1.61791600 \\ \mathrm{H} & 0.49173100 \\ \mathrm{H} & 0.88993800\end{array}$

$$
\begin{array}{r}
1.03583100 \\
-0.37877700 \\
-1.11084500 \\
-1.21278200 \\
0.17266600 \\
1.27837000 \\
-1.42177100 \\
-0.27647500 \\
-0.43825100 \\
1.01073300 \\
1.72711400 \\
-1.28595800 \\
-2.02047600 \\
0.21658600 \\
0.19917800 \\
1.34083600 \\
2.24170500
\end{array}
$$

Table S29. MP2/6-311++G(2d,2p) optimised Cartesian coordinates ( $\AA$ ) of $\mathbf{I}_{\mathrm{d}}$.
C 0.82972200
C -1.38972500
C 0.67242400
C -0.78901900
C 1.39356100
C -0.61832700
$\mathrm{H}-2.44887000$
$\mathrm{H} \quad 0.82792100$
$\mathrm{H}-0.87160200$
$\mathrm{H} \quad 0.86356900$
$\mathrm{H}-0.73658900$
H 1.47924500
$\mathrm{H}-1.29237300$
H 1.37946400
$\mathrm{H}-1.31282600$
$\mathrm{H} \quad 2.46501500$
H -0.94477400

$$
\begin{array}{rr}
-0.92053000 & -0.25406300 \\
-0.11385900 & 0.41072800 \\
0.98584900 & -0.46626700 \\
1.21205200 & -0.10215400 \\
-0.06613800 & 0.69351800 \\
-1.25630800 & -0.25689500 \\
-0.18647200 & 0.18967000 \\
0.72414000 & -1.51549600 \\
1.99198900 & 0.64868200 \\
0.15892900 & 1.60679200 \\
-2.19753000 & 0.28676000 \\
-1.32503800 & -1.02144800 \\
-0.17775300 & 1.49112700 \\
1.79299700 & -0.27816900 \\
1.55176300 & -0.99116800 \\
0.05895400 & 0.71249000 \\
-1.43837700 & -1.27843200
\end{array}
$$

Table S30. MP2/6-311++G(2d,2p) optimised Cartesian coordinates ( $\AA$ ) of $\mathbf{I}_{\text {int }}$.

$\begin{array}{lr}\text { C } & 1.37116100 \\ \text { C } & 0.73103900 \\ \text { C } & -0.59725000\end{array}$

$-0.00046200$

1.21799900

0.69701800
0.54030300

$-0.15234800$

$-0.62554600$ 


$\begin{array}{rrrr}\mathrm{C} & -1.56640800 & -0.00004800 & 0.73740200 \\ \mathrm{C} & -0.59781700 & -0.69623000 & -0.62570300 \\ \mathrm{C} & 0.73024400 & -1.21825600 & -0.15278600 \\ \mathrm{H} & -1.33436800 & 1.29399000 & -1.14408500 \\ \mathrm{H} & 0.65830900 & 2.10615300 & 0.46814800 \\ \mathrm{H} & 1.27762600 & 1.49392800 & -1.05661300 \\ \mathrm{H} & 2.45164200 & -0.00079400 & 0.45937900 \\ \mathrm{H} & 1.12622100 & -0.00054000 & 1.59996100 \\ \mathrm{H} & -1.30266500 & 0.87419400 & 1.32706500 \\ \mathrm{H} & -2.59907200 & -0.00024500 & 0.42480900 \\ \mathrm{H} & -1.30211700 & -0.87348000 & 1.32793900 \\ \mathrm{H} & -1.33498700 & -1.29250200 & -1.14495500 \\ \mathrm{H} & 0.65688800 & -2.10651300 & 0.46749100 \\ \mathrm{H} & 1.27670600 & -1.49431600 & -1.05706800\end{array}$

Table S31. MP2/6-311++G(2d,2p) optimised Cartesian coordinates ( $\AA$ ) of II.

$\begin{array}{lrrr}\mathrm{C} & 1.50411600 & 0.71848800 & -0.22061000 \\ \mathrm{C} & 0.07535000 & 1.19121600 & 0.09442000 \\ \mathrm{C} & -0.77224900 & 0.01011700 & -0.02724600 \\ \mathrm{C} & -2.22929700 & 0.00698900 & 0.00327600 \\ \mathrm{C} & 0.05836800 & -1.17971900 & -0.13484200 \\ \mathrm{C} & 1.47838700 & -0.74031600 & 0.25349700 \\ \mathrm{H} & 0.02760500 & -1.30340700 & -1.24064100 \\ \mathrm{H} & -0.00151300 & 1.36978000 & 1.18789600 \\ \mathrm{H} & -0.31414300 & 2.08943700 & -0.37808200 \\ \mathrm{H} & 1.67451000 & 0.77049200 & -1.29457600 \\ \mathrm{H} & 2.25713100 & 1.32170700 & 0.27237000 \\ \mathrm{H} & -2.66969500 & 0.94662700 & -0.31020000 \\ \mathrm{H} & -2.65018400 & -0.84697800 & -0.52028300 \\ \mathrm{H} & -2.47805400 & -0.14019000 & 1.06565000 \\ \mathrm{H} & -0.36861200 & -2.09148700 & 0.27567900 \\ \mathrm{H} & 1.59305300 & -0.79793000 & 1.33461600 \\ \mathrm{H} & 2.24184600 & -1.35870300 & -0.20340600\end{array}$

Table S32. MP2/6-311++G(2d,2p) optimised Cartesian coordinates ( $\AA$ ) of $\mathrm{TS}_{\mathbf{I} \rightarrow \mathfrak{I} \mathbf{p}}$.

C 1.25741700

C 1.26122400

C -0.00327400

C -1.26480400

C -1.25381400

C $\quad 0.00286500$

$\mathrm{H}-0.00562600$

H 1.32310700

$\mathrm{H} \quad 2.10366000$

H 1.31882100

H 2.16230600

$\mathrm{H}-1.32653300$

$\mathrm{H}-2.10996800$

$\mathrm{H}-2.15744000$

$\mathrm{H}-1.31331500$

H 0.00203200

H 0.00526700
$-0.71850900$

0.79610700

1.43095300

0.79061500

$-0.72359000$

$-1.40802900$

2.42696600

0.82388700

1. 35552200

$-0.78766200$

$-1.15902300$

0.81489200

1.34676800

$-1.16832500$

$-0.79173300$

$-1.42160000$

- 2.44497100
-0.24457200
0.14490400
-0.13594300
0.14622700
-0.24618900
0.26960600
-0.57373100
1.24787100
-0.24518700
-1.32901800
0.16185100
1.24901800
-0.24258300
0.15848700
-1.33078000
1.36009800
-0.06021700

Table S33. MP2/6-311++G(2d,2p) optimised Cartesian coordinates ( $)$ of $\mathrm{TS}_{\mathbf{I f} \rightarrow \mathbf{I} \mathbf{d}}$.

C -0.06947100

C -1.12172600
$-1.38402800$

0.74503600
$-0.28261100$

0.43673800 


$\begin{array}{lrrr}\text { C } & 1.35271100 & 0.63971400 & -0.10990000 \\ \mathrm{C} & 0.04358300 & 1.41180300 & -0.30656000 \\ \mathrm{C} & 1.11896400 & -0.88197500 & 0.32402800 \\ \mathrm{C} & -1.30167400 & -0.64538500 & -0.15103600 \\ \mathrm{H} & -2.03999000 & 1.31237000 & 0.32591900 \\ \mathrm{H} & 1.94812200 & 0.61616400 & -1.01620400 \\ \mathrm{H} & 0.17101700 & 2.43632000 & 0.02844800 \\ \mathrm{H} & 0.85584200 & -0.78392900 & 1.38956900 \\ \mathrm{H} & -1.93908400 & -1.31624400 & 0.46040400 \\ \mathrm{H} & -0.07342900 & -2.31409700 & -0.84559500 \\ \mathrm{H} & -0.91078300 & 0.68805900 & 1.50467100 \\ \mathrm{H} & 1.95837600 & 1.06606800 & 0.68222900 \\ \mathrm{H} & -0.20694700 & 1.45107500 & -1.36604800 \\ \mathrm{H} & 1.99711800 & -1.50245400 & 0.20987900 \\ \mathrm{H} & -1.82553600 & -0.66798100 & -1.11512600\end{array}$

Table S34. MP2/6-311++G(2d,2p) optimised Cartesian coordinates ( $\AA$ ) of $\mathrm{TS}_{\mathrm{I}_{\mathrm{p} \rightarrow \mathrm{Id}} \text {. }}$

$\begin{array}{lrrr}\mathrm{C} & -1.51388700 & -0.03892600 & 0.16682600 \\ \mathrm{C} & -0.62698400 & -1.30431700 & -0.07643800 \\ \mathrm{C} & 0.80484600 & -0.95250900 & -0.24589800 \\ \mathrm{C} & 1.46714800 & -0.07638400 & 0.61355200 \\ \mathrm{C} & 0.67573500 & 0.95774800 & -0.50469000 \\ \mathrm{C} & -0.68822400 & 1.26233100 & 0.07032000 \\ \mathrm{H} & 1.37459900 & -1.38177300 & -1.06140800 \\ \mathrm{H} & -0.67939300 & -1.96629700 & 0.78991300 \\ \mathrm{H} & -0.97124800 & -1.86753800 & -0.93618600 \\ \mathrm{H} & -2.29627600 & -0.04098700 & -0.58431300 \\ \mathrm{H} & -2.00453000 & -0.10450900 & 1.13089800 \\ \mathrm{H} & 1.02310200 & 0.17428200 & 1.56562900 \\ \mathrm{H} & 2.53841500 & 0.02526400 & 0.53532900 \\ \mathrm{H} & 1.43723300 & 1.73558800 & -0.49703800 \\ \mathrm{H} & 0.62429600 & 0.62402300 & -1.54605100 \\ \mathrm{H} & -0.57471700 & 1.73516200 & 1.04121700 \\ \mathrm{H} & -1.18327800 & 1.97911700 & -0.58001800\end{array}$

Table S35. MP2/6-311++G(2d,2p) optimised Cartesian coordinates ( $\AA$ ) of $\mathrm{TS}_{\mathrm{I}_{\mathrm{p}} \rightarrow \mathrm{Int}}$.

$\begin{array}{rrrr}\mathrm{C} & -0.56047000 & 0.81130200 & -0.48599800 \\ \mathrm{C} & -1.69247200 & -0.10132800 & 0.47936100 \\ \mathrm{C} & -0.68841300 & -0.68503600 & -0.47201000 \\ \mathrm{C} & 0.60888000 & -1.24442800 & 0.11913400 \\ \mathrm{C} & 1.60857000 & -0.08041800 & 0.01068100 \\ \mathrm{C} & 0.75352700 & 1.18444200 & 0.19573500 \\ \mathrm{H} & -1.12466500 & -1.14873100 & -1.34210300 \\ \mathrm{H} & -1.45811500 & -0.17347300 & 1.53399800 \\ \mathrm{H} & -2.73091300 & -0.09820500 & 0.17613600 \\ \mathrm{H} & -1.00946000 & 1.36998200 & -1.29738400 \\ \mathrm{H} & -1.47398300 & 1.14150800 & 0.38292800 \\ \mathrm{H} & 0.45822900 & -1.54173200 & 1.15478900 \\ \mathrm{H} & 0.93183000 & -2.12308700 & -0.42834200 \\ \mathrm{H} & 2.41433000 & -0.14149300 & 0.73469700 \\ \mathrm{H} & 2.05087500 & -0.07119100 & -0.98315300 \\ \mathrm{H} & 0.59515400 & 1.40317400 & 1.25050300 \\ \mathrm{H} & 1.16899200 & 2.07604400 & -0.26348000\end{array}$

Table S36. MP2/6-311++G(2d,2p) optimised Cartesian coordinates $(\AA)$ of $\mathrm{TS}_{\mathrm{Id} \rightarrow \text { Int }}$. 


$\begin{array}{rrrr}\text { C } & 0.77545400 & -0.64723100 & -0.48579000 \\ \mathrm{C} & -1.38392000 & -0.15115900 & 0.48508300 \\ \mathrm{C} & 0.60829600 & 0.89519400 & -0.41913500 \\ \mathrm{C} & -0.86672900 & 1.15881100 & -0.12261800 \\ \mathrm{C} & 1.50392100 & -0.13933200 & 0.67253700 \\ \mathrm{C} & -0.60532800 & -1.24428600 & -0.25741600 \\ \mathrm{H} & -2.45719800 & -0.25346600 & 0.36696700 \\ \mathrm{H} & 1.19316700 & 1.44543100 & -1.14484900 \\ \mathrm{H} & -1.03312300 & 2.03607900 & 0.49387900 \\ \mathrm{H} & 1.06696200 & -0.22186900 & 1.65862000 \\ \mathrm{H} & -0.55593200 & -2.18504600 & 0.28296500 \\ \mathrm{H} & 1.38880700 & -1.00149200 & -1.29940000 \\ \mathrm{H} & -1.18195800 & -0.18197900 & 1.55408400 \\ \mathrm{H} & 1.19151500 & 1.24082600 & 0.56944900 \\ \mathrm{H} & -1.32400200 & 1.33670600 & -1.09516200 \\ \mathrm{H} & 2.57498500 & -0.00715900 & 0.60617700 \\ \mathrm{H} & -1.05339000 & -1.44001300 & -1.22869500\end{array}$

Table S37. MP2/6-311++G(2d,2p) optimised Cartesian coordinates ( $\AA$ ) of $\mathrm{TS}_{\text {Iint } \rightarrow \text { II }}$.
C 1.46328800
$-0.71940600$
$-0.31715400$
C 0.11764000
$-1.20378500$
0.27228400
C -0.77258200
0.05381100
0.32569400
C -2.17980600
0.01683100
$-0.29998700$
1.16647100
0.06282600
0.79546100
$-0.03881200$
$\mathrm{H}-0.95211800$
H -0.34273100
0.38329600
1.39138800
$-1.97297500$
$-0.33903100$
$-1.61463400$
1. 26959200
$-1.22451500$
0.11427500
$-0.87953600$
$-1.39162600$
$-0.74392000$
0.19942300
0.97231000
$-0.21157400$
$-0.24671300$
$-1.34919600$
2.19115000
0.03547700
1. 44371500
$-0.71084200$
H 2.04230700
1.03551700
0.96300500

Table S38. PBE/6-311++G(2d,2p) optimised Cartesian coordinates ( $(\AA)$ of $\mathbf{I}_{\mathrm{f}}$.

$\begin{array}{rrrr}\text { C } & 1.26995800 & -0.74189500 & -0.22027200 \\ \text { C } & 1.27182300 & 0.77077900 & 0.00594500 \\ \text { C } & 0.00000000 & 1.44176500 & 0.04291800 \\ \text { C } & -1.27182400 & 0.77077900 & 0.00594500 \\ \text { C } & -1.26995800 & -0.74189600 & -0.22027100 \\ \text { C } & 0.00000100 & -1.39371500 & 0.34194800 \\ \text { H } & 0.00000000 & 2.53364800 & 0.15581500 \\ \text { H } & 1.72201700 & 1.06553800 & 0.99326700 \\ \text { H } & 1.95849000 & 1.35355300 & -0.64729200 \\ \text { H } & 1.34762800 & -0.93494300 & -1.30110000 \\ \text { H } & 2.17011400 & -1.17689300 & 0.23167700 \\ \text { H } & -1.72202300 & 1.06554100 & 0.99326300 \\ \text { H } & -1.95848700 & 1.35355200 & -0.64729700 \\ \text { H } & -2.17011300 & -1.17689400 & 0.23168000 \\ \text { H } & -1.34763000 & -0.93494500 & -1.30109800 \\ \text { H } & 0.00000100 & -1.31702900 & 1.44222200 \\ \text { H } & 0.00000100 & -2.46602600 & 0.11157800\end{array}$


Table S39. PBE/6-311++G(2d,2p) optimised Cartesian coordinates ( $\mathrm{A})$ of $\mathbf{I}_{\mathrm{p}}$.

$$
\begin{array}{lr}
\mathrm{C} & -1.25366100 \\
\mathrm{C} & -1.25010500 \\
\mathrm{C} & 0.00082800 \\
\mathrm{C} & 1.25120700 \\
\mathrm{C} & 1.25269100 \\
\mathrm{C} & -0.00085300 \\
\mathrm{H} & 0.00112500 \\
\mathrm{H} & -1.26540700 \\
\mathrm{H} & -2.13355100 \\
\mathrm{H} & -1.33508300 \\
\mathrm{H} & -2.18872800 \\
\mathrm{H} & 1.26643700 \\
\mathrm{H} & 2.13531000 \\
\mathrm{H} & 2.18737200 \\
\mathrm{H} & 1.33393200 \\
\mathrm{H} & -0.00082600 \\
\mathrm{H} & -0.00145400
\end{array}
$$

$$
\begin{array}{r}
0.68937900 \\
-0.86657700 \\
-1.27819300 \\
-0.86513700 \\
0.69081000 \\
1.39462400 \\
-1.68973500 \\
-0.83682200 \\
-1.35594600 \\
0.70751000 \\
1.08924500 \\
-0.83518700 \\
-1.35336600 \\
1.09178800 \\
0.70893000 \\
1.47747200 \\
2.42557300
\end{array}
$$$$
-0.24262600
$$$$
0.27005600
$$$$
-0.29521500
$$$$
0.27015400
$$$$
-0.24277300
$$$$
0.24552700
$$$$
-1.31235400
$$$$
\text { 1. } 36535200
$$$$
-0.15197200
$$$$
-1.33749600
$$$$
0.17265200
$$$$
\text { 1. } 36544200
$$$$
-0.15181000
$$$$
0.17231300
$$$$
-1.33765700
$$$$
\text { 1. } 34200300
$$$$
-0.14755700
$$

Table S40. PBE/6-311++G(2d,2p) optimised Cartesian coordinates ( $\AA$ ) of $\mathbf{I}_{\mathrm{d}}$.

$\begin{array}{rrrr}\mathrm{C} & 0.78085600 & -1.02389600 & -0.22875300 \\ \mathrm{C} & -1.40751000 & -0.04637400 & 0.38400700 \\ \mathrm{C} & 0.74143600 & 0.99918800 & -0.43019800 \\ \mathrm{C} & -0.72869300 & 1.25779200 & -0.11319500 \\ \mathrm{C} & 1.38566600 & -0.09467500 & 0.65029300 \\ \mathrm{C} & -0.67370800 & -1.24827300 & -0.24049500 \\ \mathrm{H} & -2.47108300 & -0.07744200 & 0.12350600 \\ \mathrm{H} & 0.90707400 & 0.72265000 & -1.48726900 \\ \mathrm{H} & -0.81684700 & 2.04035400 & 0.65109200 \\ \mathrm{H} & 0.90804600 & 0.11938000 & 1.60889500 \\ \mathrm{H} & -0.85231400 & -2.16676500 & 0.35754300 \\ \mathrm{H} & 1.40726100 & -1.50355000 & -0.98890200 \\ \mathrm{H} & -1.35856500 & -0.10218000 & 1.47917400 \\ \mathrm{H} & 1.44760600 & 1.82818000 & -0.26502100 \\ \mathrm{H} & -1.22375800 & 1.62849900 & -1.01967500 \\ \mathrm{H} & 2.47508600 & -0.05144100 & 0.66730900 \\ \mathrm{H} & -1.01079100 & -1.50025700 & -1.25660500\end{array}$

Table S41. PBE/6-311++G(2d,2p) optimised Cartesian coordinates ( $(\AA)$ of $\mathbf{I}_{\text {int }}$.

$\begin{array}{lrrr}\text { C } & 1.38921600 & -0.25544700 & 0.51696200 \\ \text { C } & 0.93806900 & 1.12530300 & -0.01111100 \\ \text { C } & -0.41885800 & 0.90383500 & -0.55689100 \\ \text { C } & -1.62367200 & -0.01641500 & 0.70076500 \\ \text { C } & -0.73435700 & -0.47956100 & -0.62506400 \\ \text { C } & 0.52229600 & -1.26850200 & -0.26757900 \\ \mathrm{H} & -1.05624800 & 1.69404900 & -0.96181000 \\ \mathrm{H} & 1.03647200 & 1.97640700 & 0.67514500 \\ \mathrm{H} & 1.51028600 & 1.41727200 & -0.91991500 \\ \mathrm{H} & 2.46177600 & -0.42320900 & 0.37864300 \\ \mathrm{H} & 1.19394800 & -0.31869400 & 1.59596400 \\ \mathrm{H} & -1.47222000 & 0.98559600 & 1.15064100 \\ \mathrm{H} & -2.67143700 & -0.07149800 & 0.39990400 \\ \mathrm{H} & -1.32236600 & -0.72864800 & 1.47490300 \\ \mathrm{H} & -1.43829000 & -0.86090400 & -1.36572100 \\ \mathrm{H} & 0.30689400 & -2.18976700 & 0.28616500 \\ \mathrm{H} & 1.00497200 & -1.56273700 & -1.21137800\end{array}$


Table S42. PBE/6-311++G(2d,2p) optimised Cartesian coordinates ( $(\AA)$ of II.
C -1.48995800
- 0.74868200
0.23670000
C -0.05686000
$-1.18608700$
$-0.12808900$
C 0.77871000
0.00916300
$-0.02433000$
C -0.07193400
C -1.51526300
$\mathrm{H}-2.25203900$
$\mathrm{H}-1.63304900$
$\mathrm{H}-0.00901200$
1.19638000
0.08122800
0.72823800
$-0.19931700$
$-1.36195800$
- 0.25406000
- 0.84322400
1. 32278400
$-1.37675700$
$-1.23286900$
$-2.09759100$
0.31807500
1. 45825200
1. 16564100
2. 08478300
1. 32713200
$-0.43907600$
0.33277400
0.81776700
$-1.27538800$
0.00818400
0.00465700
0.96392800
$-0.28385900$
$-0.17549600$
1. 06537300
$\mathrm{H} 2.51739700$
$-0.84001300$
$-0.54448800$

Table S43. PBE/6-311++G(2d,2p) optimised Cartesian coordinates $(\AA)$ of $\mathrm{TS}_{\mathrm{If} \rightarrow \mathrm{Ip}}$.

C 1.26685600

C 1.26738200

C -0.00002100

C -1.26740600

C -1.26683400

C $\quad 0.00002100$

$\mathrm{H}-0.00003600$

$\mathrm{H} \quad 1.37444700$

H 2.11719500

$\mathrm{H} \quad 1.36521200$

$\mathrm{H} \quad 2.17096400$

$\mathrm{H}-1.37447200$

$\mathrm{H}-2.11723400$

$\mathrm{H}-2.17092900$

$\mathrm{H}-1.36518600$

$\mathrm{H} \quad 0.00002100$

H $\quad 0.00003700$
$-0.72759400$

0.80878300

1.44285600

0.80874500

$-0.72763200$

$-1.42031400$

2.43300100

0.82629000

1.36469500

$-0.78751400$

$-1.16946400$

0.82624700

1.36463300

$-1.16952800$

$-0.78755500$

$-1.49651200$

$-2.45610900$
$-0.23270400$

0.14588500

$-0.13027200$

0.14588400

$-0.23270600$

0.25106200

$-0.60544800$

1.25511000

$-0.26493400$

$-1.32570600$

0.20192900

1.25510900

$-0.26493500$

0.20192500

$-1.32570900$

1.34931900

$-0.12542100$

Table S44. PBE/6-311++G(2d,2p) optimised Cartesian coordinates ( $\AA$ ) of $\mathrm{TS}_{\mathrm{If} \rightarrow \mathrm{Id}}$.

$\begin{array}{lr}\text { C } & 0.11662600 \\ \text { C } & -1.23048800 \\ \text { C } & 1.25580400 \\ \text { C } & -0.11316800 \\ \text { C } & 1.20391700 \\ \text { C } & -1.20066400 \\ \text { H } & -2.21485500 \\ \text { H } & 1.68745200 \\ \text { H } & -0.05139800 \\ \text { H } & 0.93025800 \\ \text { H } & -1.83346100 \\ \text { H } & 0.27355600 \\ \text { H } & -1.07682500 \\ \text { H } & 1.98879900 \\ \text { H } & -0.40183800\end{array}$

$-1.40095500$

0.59239300

0.74516000

1.44395000

$-0.74848000$

$-0.80511200$

1.05016300

0.64668400

2.40623300

$-0.53741500$

$-1.53373400$

$-2.30425500$

0.54057300

1.25610200

1.66219200
-0.23094700
0.41724000
-0.21820200
-0.23355700
0.42483200
-0.18971300
0.26642100
-1.21980200
0.28979900
1.47578500
0.37824800
-0.83348100
1.50617400
0.41809100
-1.27087200 


$\begin{array}{lrrr}\mathrm{H} & 2.16883600 & -1.25721000 & 0.36712200 \\ \mathrm{H} & -1.66268500 & -0.89107200 & -1.19540100\end{array}$

Table S45. PBE/6-311++G(2d,2p) optimised Cartesian coordinates $(\AA)$ of $\mathrm{TS}_{\mathrm{Ip} \rightarrow \mathrm{Id}}$.

$\begin{array}{lr}\text { C } & -1.51379100 \\ \text { C } & -0.70096300 \\ \text { C } & 0.74216200 \\ \text { C } & 1.45006900 \\ \text { C } & 0.75976100 \\ \text { C } & -0.62203700 \\ \text { H } & 1.28921500 \\ \text { H } & -0.81493700 \\ \text { H } & -1.08870500 \\ \text { H } & -2.29923100 \\ \text { H } & -2.02509200 \\ \text { H } & 1.06700900 \\ \text { H } & 2.53799100 \\ \text { H } & 1.52072900 \\ \text { H } & 0.73422600 \\ \text { H } & -0.52731100 \\ \text { H } & -1.08510500\end{array}$

0.04210000
-1.28826100
-1.06139900
-0.12577100
0.96953200
1.30567900
-1.56246000
-1.92514100
-1.86601800
0.06172100
0.00852300
0.11958500
-0.12452200
1.76492900
0.64191900
1.79400000
2.03617800

0.15397000

$-0.07897300$

$-0.21840500$

0.57322600

$-0.46768500$

0.05747500

$-1.02483200$

0.82109800

$-0.92448700$

$-0.61061900$

1.12111500

1.56625800

0.49431300

$-0.45094000$

$-1.52440600$

1.03576900

$-0.62091300$

Table S46. PBE/6-311++G(2d,2p) optimised Cartesian coordinates $(\AA)$ of $\mathrm{TS}_{\mathrm{Ip} \rightarrow \mathbf{I i n t}}$.
C -0.58107200
0.83006400
$-0.45068400$
C -1.71628400
$-0.14302900$
0.48197400
C -0.70300100
$-0.69509800$
$-0.45452800$
$-1.25169700$
0.11427700
$-0.08163300$
0.01625900
C 1.61449700
C 0.76266900
1.19418500
0.18961800
$-1.13672000$
$-1.36088400$
$-0.19033300$
1.55460800
$-0.09943400$
0.16820000
1. 36430300
$-1.29952100$
1.17455800
0.38574700
$-1.57770300$
1.15442800
$-2.12956500$
$-0.45525600$
$-0.14577600$
0.75876300
$-0.07472200$
$-0.97597700$
1.45983000
1.24921200
2. 08010100
$-0.31074300$

Table S47. PBE/6-311++G(2d,2p) optimised Cartesian coordinates $(\AA)$ of $\mathrm{TS}_{\mathrm{Id} \rightarrow \text { Iint }}$.
C 0.77940000
$-0.65200900$
$-0.47918800$
C -1.40834300
$-0.15490100$
0.47063000
C 0.60740800
0.89959300
$-0.41643800$
C -0.86821900
1.16606400
$-0.10660900$
$-0.14620300$
0.66861400
C -0.60600000
$-1.25456100$
$-0.24925200$
$-0.25525500$
0.31701600
1.17915600
1. 44504900
2.03988100
$-1.17246000$
0.53453900
$-0.21672600$
1.67382800
$-2.19658700$
0.31058100
$-1.00975000$
$-1.31310100$
$\mathrm{H} \quad 1.38296900$
$\mathrm{H}-1.24813000$
$-0.19323300$
1.55778900 


$\begin{array}{rrrr}\mathrm{H} & 1.21997100 & 1.26909000 & 0.55344700 \\ \mathrm{H} & -1.32503700 & 1.38733400 & -1.08369400 \\ \mathrm{H} & 2.61427800 & -0.01139700 & 0.58633300 \\ \mathrm{H} & -1.04374800 & -1.48210300 & -1.23039100\end{array}$

Table S48. PBE/6-311++G(2d,2p) optimised Cartesian coordinates ( $(\AA)$ of $\mathrm{TS}_{\text {Iint } \rightarrow \text { II }}$.

$\begin{array}{rrrr}\mathrm{C} & -1.51322000 & -0.69493400 & 0.25107900 \\ \mathrm{C} & -0.12988100 & -1.23309900 & -0.22365000 \\ \mathrm{C} & 0.78113800 & 0.00764600 & -0.40111100 \\ \mathrm{C} & 2.15347900 & 0.02675400 & 0.35819100 \\ \mathrm{C} & -0.04871700 & 1.15985900 & -0.17340500 \\ \mathrm{C} & -1.42122800 & 0.83909700 & 0.11878500 \\ \mathrm{H} & 1.09658400 & 0.16634500 & -1.46748300 \\ \mathrm{H} & 0.30696800 & -1.92501600 & 0.50561700 \\ \mathrm{H} & -0.21408100 & -1.78160700 & -1.16905200 \\ \mathrm{H} & -2.33971600 & -1.09861900 & -0.34265800 \\ \mathrm{H} & -1.71171000 & -0.96378800 & 1.29525600 \\ \mathrm{H} & 2.74687100 & -0.81958100 & -0.00304000 \\ \mathrm{H} & 2.70929300 & 0.95409300 & 0.17855400 \\ \mathrm{H} & 1.97294700 & -0.09129900 & 1.43273300 \\ \mathrm{H} & 0.32586200 & 2.18780700 & -0.25261000 \\ \mathrm{H} & -1.80160100 & 1.45673400 & 0.95994800 \\ \mathrm{H} & -2.02084400 & 1.28299700 & -0.71660300\end{array}$

Table S49. QCISD(T)/CBS(MP2)//method ${ }^{\mathrm{a}}$ calculated energies (Hartrees).

\begin{tabular}{lllll}
\hline method & QCISD & PBE & B 3 LP & MP2 \\
\hline $\mathbf{I}_{\mathrm{f}}$ & -234.5999252 & -234.598120 & -234.599509 & -234.599938 \\
$\mathbf{I}_{\mathrm{p}}$ & -234.6060849 & -234.605752 & -234.606231 & -234.606162 \\
$\mathbf{I}_{\mathrm{d}}$ & -234.6026795 & -234.601506 & -234.601328 & -234.602707 \\
$\mathrm{TS}_{\mathbf{I f} \rightarrow \mathbf{I}_{\mathrm{p}}}$ & -234.5964963 & -234.595766 & -234.596698 & -234.596910 \\
$\mathrm{TS}_{\mathbf{I f} \rightarrow \mathbf{I d}}$ & -234.5956952 & -234.595321 & -234.596096 & -234.598743 \\
$\mathrm{TS}_{\mathbf{I}_{\mathrm{p} \rightarrow \mathbf{I d}}}$ & -234.5984222 & -234.597636 & -234.597695 & -234.598748 \\
$\mathrm{TS}_{\mathbf{I}_{\mathrm{p} \rightarrow \mathbf{I n t}}}$ & -234.5865696 & -234.586075 & -234.586583 & -234.587157 \\
$\mathrm{TS}_{\mathbf{I d} \rightarrow \mathbf{I n t}}$ & -234.5918567 & -234.591382 & -234.591985 & -234.592247 \\
$\mathbf{I}_{\text {int }}$ & -234.6023834 & -234.601415 & -234.599914 & -234.602752 \\
$\mathrm{TS}_{\mathbf{I i n t} \rightarrow \mathbf{I I}}$ & -234.5911046 & -234.590299 & -234.591795 & -234.591492 \\
$\mathbf{I I}$ & -234.6194647 & -234.618443 & -234.619169 & -234.619436 \\
\hline
\end{tabular}

${ }^{\mathrm{a} D F T}$ methods used 6-311++G(2d,2p) basis sets. QCISD used 6-311++G(d,p) basis sets. 
Table S50. QCISD(T)/CBS(QCISD)//QCISD/6-311++G(d,p) calculated energies (Hartrees).

\begin{tabular}{|c|c|c|c|c|c|}
\hline method & QCISD(T)/DZ & $\mathrm{QCISD} / \mathrm{DZ}^{\mathrm{a}}$ & $\mathrm{QCISD} \mathrm{TZ}^{\mathrm{b}}$ & 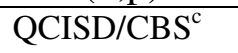 & $\mathrm{QCISD}(\mathrm{T})+\Delta \mathrm{QCISD}$ \\
\hline $\mathbf{I}_{\mathrm{f}}$ & -234.304667 & -234.272827 & -234.477260 & -234.549203 & -234.581043 \\
\hline $\mathbf{I}_{\mathrm{p}}$ & -234.312624 & -234.278528 & -234.481036 & -234.552301 & -234.586397 \\
\hline $\mathbf{I}_{\mathrm{d}}$ & -234.306262 & -234.272205 & -234.476764 & -234.548750 & -234.582807 \\
\hline $\mathrm{TS}_{\mathbf{I} \boldsymbol{f} \rightarrow \mathbf{I} \mathrm{p}}$ & -234.303371 & -234.270945 & -234.473492 & -234.544772 & -234.577198 \\
\hline $\mathrm{TS}_{\mathbf{I f} \rightarrow \mathbf{I d}}$ & -234.302129 & -234.269207 & -234.471996 & -234.543360 & -234.576283 \\
\hline $\mathrm{TS}_{\mathbf{I}_{\mathrm{p} \rightarrow \mathbf{I d}}}$ & -234.303940 & -234.269639 & -234.472880 & -234.544404 & -234.578705 \\
\hline $\mathrm{TS}_{\mathrm{I}_{\mathrm{p} \rightarrow \mathrm{Iint}}}$ & -234.291454 & -234.257195 & -234.460753 & -234.532388 & -234.566647 \\
\hline $\mathrm{TS}_{\mathbf{I}_{\mathbf{d} \rightarrow \mathbf{I i n t}}}$ & -234.296763 & -234.262380 & -234.465955 & -234.537596 & -234.571979 \\
\hline $\mathbf{I}_{\text {int }}$ & -234.307335 & -234.273118 & -234.476664 & -234.548294 & -234.582511 \\
\hline $\mathrm{TS}_{\text {Iint } \rightarrow \text { II }}$ & -234.297720 & -234.265227 & -234.467990 & -234.539346 & -234.571838 \\
\hline II & -234.325243 & -234.293006 & -234.496552 & -234.568183 & -234.600420 \\
\hline
\end{tabular}

a aug-cc-pVDZ basis set. ${ }^{b}$ aug-cc-pVTZ basis set. ${ }^{c}$ see Refs. 19 and 20 of main text. 


\section{Details of the kinetic modelling of the carbocation rearrangement of cyclohexylium to methylcyclopentylium.}

As an illustrative example the elementary steps identified by QCISD in the transformation of cyclohexylium to methylcyclopentylium are given below.

1) $I_{f} \leftrightarrow I_{p}$

2) $I_{f} \leftrightarrow I_{d}$

3) $I_{p} \leftrightarrow I_{d}$

4) $I_{p} \leftrightarrow I_{\text {int }}$

5) $I_{d} \leftrightarrow I_{\text {int }}$

6) $I_{\text {int }} \leftrightarrow$ II

Let $k_{i}$ and $k_{-i}$ be the forward and reverse rate constants for the elementary step $i$, respectively and $r_{i}$ be the net rate of elementary step $i$. For example, the net rate of elementary step (1) is given by

$$
r_{1}=k_{1} C_{I_{f}}-k_{-1} C_{I_{d}}
$$

The rate constants and the concentrations are expressed in the units $\mathrm{s}^{-1}$ and $\mathrm{gmol} / \mathrm{cm}^{3}$ respectively. A kinetic model is derived by considering all the elementary steps occurring in a batch reactor with ideal gas assumption and the resulting set of coupled ordinary differential equations are solved to obtain the concentration profiles of all five species with respect to time. The time domain solution of the reactor system reveals that the concentration of species $I_{p}$ and $I_{\text {int }}$ are very small as compared to other species and species $\mathbf{I}_{\mathbf{f}}$ and $\mathbf{I}_{\mathrm{p}}$ reach equilibrium concentrations as dictated by elementary step (1) before being consumed to form the product and other intermediates. It is important to realise that species $I_{d}$ and $I_{\text {int }}$ are Bodenstein intermediates for which the quasi steady-state approximation (QSSA) can be applied as given below.

$$
\frac{d C_{I_{f}}}{d t}=\frac{d C_{I_{d}}}{d t}=0
$$

Eq. (2) leads to the following algebraic constraints on reaction rates. 


$$
\begin{aligned}
& r_{2}+r_{3}-r_{5}=0 \\
& r_{4}+r_{5}-r_{6}=0
\end{aligned}
$$

Simultaneous solution of eqs. (3) and (4) yields expressions for unknown concentrations $C_{1 \mathrm{c}}$ and $C_{\mathrm{l} 2 \mathrm{a}}$ in terms of measurable concentrations of species $\mathbf{I}_{\mathrm{f}}, \mathbf{I}_{\mathrm{p}}$, and II as given below.

$$
\begin{aligned}
& C_{I_{d}}=d_{1} C_{I_{f}}+d_{2} C_{I_{p}}+d_{3} C_{I I} \\
& C_{I_{\mathrm{int}}}=d_{4} C_{I_{f}}+d_{5} C_{I_{p}}+d_{6} C_{I I}
\end{aligned}
$$

The coefficients $d_{i}$ and $b_{i}$ are the functions of rate constants alone as given below.

$$
\begin{aligned}
& d_{1}=\left(b_{1}+\frac{b_{1} b_{3} b_{5}}{1-b_{3} b_{5}}\right) \\
& d_{2}=\left(b_{2}+\frac{b_{3} b_{4}+b_{2} b_{3} b_{5}}{1-b_{3} b_{5}}\right) \\
& d_{3}=\left(\frac{b_{3} b_{6}}{1-b_{3} b_{5}}\right) \\
& d_{4}=\left(\frac{b_{1} b_{5}}{1-b_{3} b_{5}}\right) \\
& d_{5}=\left(\frac{b_{4}+b_{2} b_{5}}{1-b_{3} b_{5}}\right) \\
& d_{6}=\left(\frac{b_{6}}{1-b_{3} b_{5}}\right) \\
& b_{1}=\left(\frac{k_{2}}{k_{-2}+k_{-3}+k_{5}}\right)
\end{aligned}
$$




$$
\begin{aligned}
& b_{2}=\left(\frac{k_{3}}{k_{-2}+k_{-3}+k_{5}}\right) \\
& b_{3}=\left(\frac{k_{-5}}{k_{-2}+k_{-3}+k_{5}}\right) \\
& b_{4}=\left(\frac{k_{4}}{k_{-4}+k_{-5}+k_{6}}\right) \\
& b_{5}=\left(\frac{k_{5}}{k_{-4}+k_{-5}+k_{6}}\right) \\
& b_{6}=\left(\frac{k_{-6}}{k_{-4}+k_{-5}+k_{6}}\right)
\end{aligned}
$$

The equilibrium concentrations of $I_{f}$ and $I_{p}$ as dictated by elementary step (1) are given by

$$
\begin{aligned}
C_{I_{f}}{ }^{e} & =\frac{C_{I_{f}}{ }^{0}+C_{I_{p}}{ }^{0}}{1+K_{1}} \\
C_{I_{p}}{ }^{e} & =\frac{K_{1}\left(C_{I_{f}}{ }^{0}+C_{I_{p}}{ }^{0}\right)}{1+K_{1}}
\end{aligned}
$$

where $C_{\mathrm{lf}}{ }^{0}$ and $C_{\mathrm{lp}}{ }^{0}$ are initial concentrations of species $\mathrm{I}_{\mathrm{f}}$ and $\mathrm{I}_{\mathrm{p}}$ and $K_{1}$ is the equilibrium constant for elementary step (1).

The rate expression for calculating the net rate of formation of product II is given by

$$
r=r_{6}=k_{6} C_{I_{\mathrm{int}}}-k_{-6} C_{I I}
$$

By substituting eqs. (19) and (20) into eq. (6) the net rate of formation of product II can be expressed in the following form. 


$$
r=k_{f} C_{A}-k_{r} C_{I I}
$$

where $k_{f}$ and $k_{r}$ are overall forward and reverse rate constants and given by

$$
\begin{aligned}
& k_{f}=\frac{k_{6}\left(d_{4}+d_{5} K_{1}\right)}{1+K_{1}} \\
& k_{r}=k_{-6}-d_{6}
\end{aligned}
$$

The concentration $C_{A}$ is the sum of concentrations of species $\mathrm{I}_{\mathrm{f}}$ and $\mathrm{I}$. The ratio $C_{\mathrm{lp}} / C_{\mathrm{lf}}$ is equal to the equilibrium constant $K_{1}$. To make a comparison with the Arrhenius parameters calculated by Attina et al., the overall forward rate constant $k_{f}$ (cf. eq. 23) is calculated at various temperatures and the activation energy and pre-exponential factor are derived from an Arrhenius plot. An example of this calculation is shown in Figure $\mathrm{S1}$ for the reaction profile predicted by $Q C I S D(T) / C B S / Q C I S D / 6-311++G(d, p)$. 


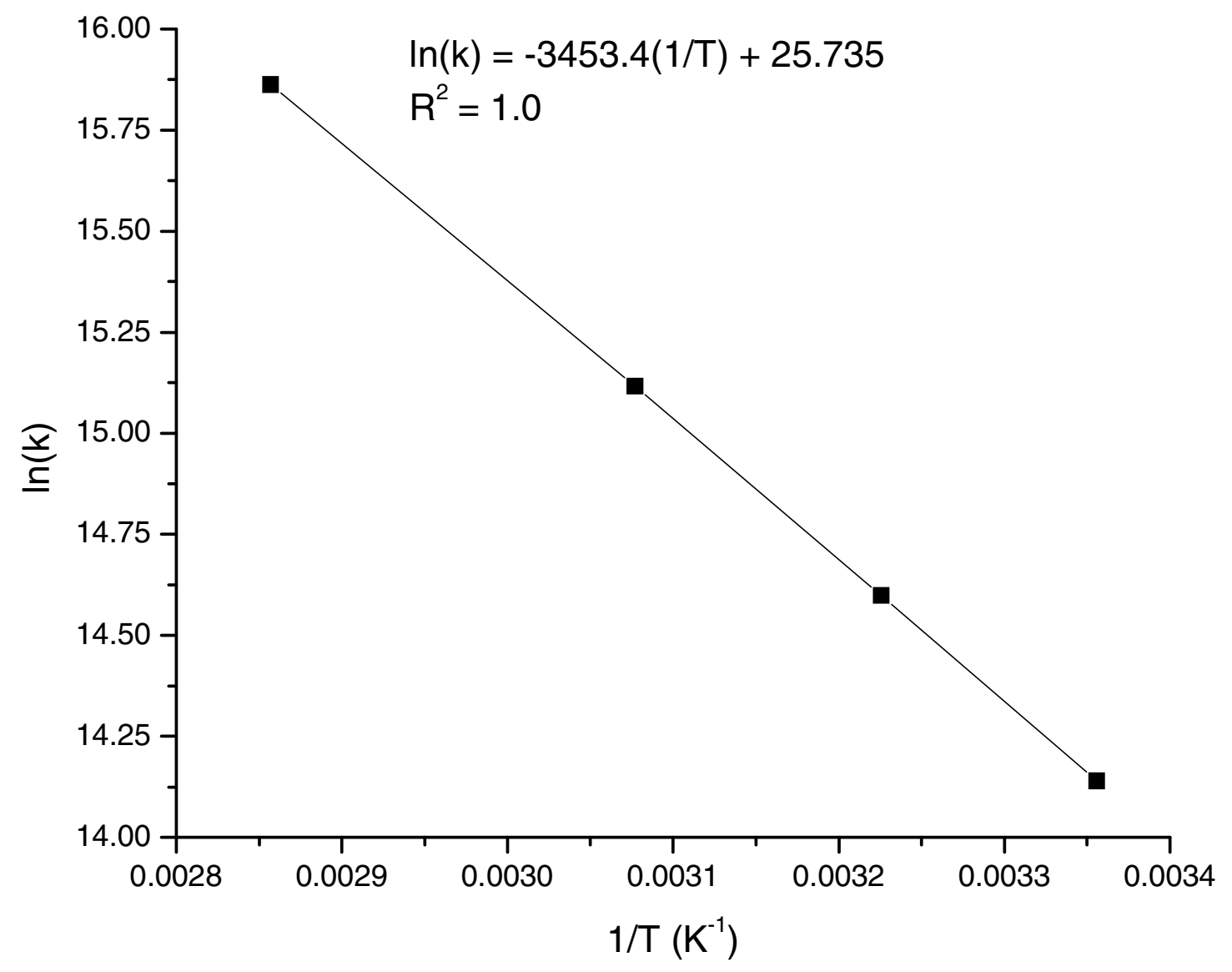

Figure S1. Calculation of activation energy and pre-exponential factor for the reaction profile predicated by $\mathrm{QCISD}(\mathrm{T}) / \mathrm{CBS} / / \mathrm{QCISD} / 6-311++\mathrm{G}(\mathrm{d}, \mathrm{p})$ (see text). $E_{a}=6.9$ $\mathrm{kcal} / \mathrm{mol}$ and $A=10^{11.18} \mathrm{~s}^{-1}$. 